\title{
Relaxations for two-level multi-item lot-sizing problems
}

\author{
Mathieu Van Vyve • Laurence A. Wolsey • \\ Hande Yaman
}

Received: 9 August 2012 / Accepted: 13 July 2013

(C) Springer-Verlag Berlin Heidelberg and Mathematical Optimization Society 2013

\begin{abstract}
We consider several variants of the two-level lot-sizing problem with one item at the upper level facing dependent demand, and multiple items or clients at the lower level, facing independent demands. We first show that under a natural cost assumption, it is sufficient to optimize over a stock-dominant relaxation. We further study the polyhedral structure of a strong relaxation of this problem involving only initial inventory variables and setup variables. We consider several variants: uncapacitated at both levels with or without start-up costs, uncapacitated at the upper level and constant capacity at the lower level, constant capacity at both levels. We finally demonstrate how the strong formulations described improve our ability to solve instances with up to several dozens of periods and a few hundred products.
\end{abstract}

Keywords Mixed-integer programming · Lot-sizing · Extended formulation · Multi-level $\cdot$ Multi-item

Mathematics Subject Classification (2000) 68Q25 - 90C11 · 90C27 - 90C35 · 90B05 $\cdot 90 \mathrm{~B} 06$

\footnotetext{
This text presents research results of the Belgian Program on Interuniversity Poles of Attraction initiated by the Belgian State, Prime Minister's Office, Science Policy Programming. The scientific responsibility is assumed by the authors. The research of the third author is supported by TUBITAK.
}

M. Van Vyve $(\varangle) \cdot$ L. A. Wolsey

CORE, voie du Roman Pays 34 bte L1.03.01, Louvain-la-Neuve 1348, Belgium

e-mail: mathieu.vanvyve@uclouvain.be

L. A. Wolsey

e-mail: laurence.wolsey@uclouvain.be

H. Yaman

Department of Industrial Engineering, Bilkent University, 06800 Bilkent, Ankara, Turkey

e-mail: hyaman@bilkent.edu.tr 


\section{Introduction}

We study two-level multi-item multi-period planning problems on a finite horizon with time-dependent demand. In this context, multi-level means that there is dependent demand in the system: some goods are consumed by the production of others. We focus on problems with one item at the upper-level facing dependent demand, and multiple items or clients at the lower level, facing independent demands. The two levels can represent different stages of a production process executed at a single location (e.g., making and packing, bulk and end products, component and assembly), but can also represent production and transportation to clients, in which case the problem is known as the one warehouse, multiple retailer (OWMR) problem. One key aspect of the models that we consider is that holding inventory is possible at both levels. We study various polyhedra related to such problems. In particular, we consider the uncapacitated problem, the problem with start-up cost at both levels, and some capacitated variants.

Our results include (i) a new "Wagner-Whitin" type relaxation of the two-level problem, (ii) a proof that this relaxation solves the original problem under certain natural cost conditions, (iii) a further decomposition as the intersection of "discrete lotsizing" relaxations for which we provide convex hull descriptions in both original space and with compact extended formulations, (iv) similar relaxations and formulations for a variant with start-ups and a variant with constant production capacities and (v) computational results for two-level lot-sizing problem with start-ups and the problem with constant production capacities based on the appropriate extended formulation.

The seminal papers of Wagner and Whitin [31] and Zangwill [32] show how to solve the uncapacitated single-level and multi-level in-series lot-sizing problems in polynomial time. Veinott [29] generalizes the approach to more general product structures leading to non-polynomial-time algorithms. van Hoesel et al. [25] give a polynomialtime algorithm for a two-level problem with constant production capacity at the upper level. Hwang [13] gives polynomial-time algorithms for uncapacitated single-item two-level problems with more general cost structures.

Several important hardness results have been proved. Bitran and Yanasse [7] show that the single-item lot-sizing problem becomes NP-Hard when the production capacity varies over time. Arkin et al. [3] show that the Joint Replenishment Problem (two levels with one item at the upper level without inventory and multiple items at the lower level) is NP-Hard. The one-level multi-item problem with a joint capacity constraint generalizes the problem of optimizing over a single-node flow set and is NP-Hard. Since most realistic problems involve at least one of these three characteristics (varying capacity, divergent product structure, joint capacity) and are therefore NP-Hard, much research in the last 30 years has been devoted to finding (provably) strong reformulations that can then be used in MIP solvers, as opposed to searching for direct optimization algorithms. The present paper follows this line of research of which Pochet and Wolsey [22] provides an in-depth survey.

For single-item lot-sizing, many polyhedral results have been obtained both for the basic uncapacitated model $[6,14]$ and for extensions including backlogging $[5,15,18]$, start-ups [27], constant capacity [20], increasing capacities [23], sales, or a combination of these [30]. These results can be classified into two categories: linear descrip- 
tion of the convex hull of solutions in the original variable space, usually of exponential size and accompanied by an efficient separation algorithm on the one hand, and tight extended formulation involving additional variables, usually of polynomial size on the other hand. For the latter, Van Vyve and Wolsey [28] show how to create and manage a trade-off between strength and size of these extended formulations.

Within this line of research Pochet and Wolsey [21] is crucial in terms of motivation. They show that the non-speculative cost assumption, which often is satisfied in practice and has been shown to translate into faster optimization algorithms [2,10,26], has an analog in polyhedral combinatorics. Specifically, under this cost assumption, to solve the problem, it suffices to optimize over the stock-dominant of the solution set, without requiring non-negativity of production. The resulting polyhedron is called the Wagner-Whitin relaxation. It has a much simpler polyhedral structure and is a very strong relaxation of the original model.

For multi-item problems, Clark and Scarf [8] introduced the concept of echelonstock. This later proved to be key in building strong single-item relaxations of multilevel models leading to efficient branch-and-bound algorithms based on Lagrangian relaxation [1] or cutting plane approaches [19]. Less progress has been made on the polyhedral structure of multi-level models beyond such single-item relaxations. The multi-commodity extended reformulation applicable to any single-source fixed-charge network flow problem is known to be very strong, but it is not tight for in-series models, even for two levels and under the non-speculative cost assumption. Melo and Wolsey [17] give a tight $\mathcal{O}\left(n^{3}\right)$ formulation of the uncapacitated two-level in-series model. Zhang et al. [33] give a partial description of the convex hull of solutions in the original variable space for the same model, allowing also for intermediate independent demand.

To the best of our knowledge, no polyhedral work has been done for multi-level lot-sizing models involving start-ups, capacities, or multiple items at the lower level (beyond single-item relaxations based on the echelon-stock concept). The present work partially fills this gap. Following Pochet and Wolsey [21], we consider stockdominant relaxations of these multi-level problems that we prove are sufficient to solve the problem under specific cost assumptions.

The rest of the paper is organized as follows. In Sect. 2 we describe the capacitated two-level lot-sizing model $2 L S$, its stock-dominant relaxation $2 W W$ and the closely related two-level discrete lot-sizing problem $2 D L S$, whose polyhedral structure we study in order to obtain a good formulation for $2 W W$. We prove that solving $2 W W$ solves $2 L S$ under a natural "non-speculative" cost assumption. Section 3 is devoted to the polyhedral analysis of several variants of $2 D L S$. In Sect. 3.1 we consider the basic uncapacitated $2 D L S-(U, U)$ model and give a polynomial-size linear programming (LP) extended formulation, together with its projection onto the original variable space. The next subsections extend, sometimes partially, these results in several directions. In Sect. 3.2 we consider the model $2 D L S-(U, U)-S C$ that includes start-ups and extend the result obtained for $2 D L S-(U, U)$. In Sects. 3.3 and 3.4 we derive results for the case with constant capacity limits on production of items at the lower level, and at both levels, respectively. In Sect. 4 we demonstrate how these strong formulations improve our ability to solve several variants of two-level planning problems. We also indicate 
what may be the best modeling options for instances of very large size. We conclude in the last section by discussing some open problems.

\section{The two-level multi-item lot sizing problem and its Wagner-Whitin relaxation}

Here we present the problem of interest and the non-speculative relaxations that we will study.

Let $n$ be the length of the planning horizon, $I$ be the set of items at the lower level with $m=|I|$ and 0 be the item at the upper level. We define $I_{0}=I \cup\{0\}$. For integers $a$ and $b$, we use $[a, b]$ to denote the set of integers $\{a, \ldots, b\}$ from $a$ to $b$. We denote the demand in period $j \in[1, n]$ for item $i \in I$ by $d_{j}^{i}$ and the setup, production, inventory holding costs and the capacity for item $i \in I_{0}$ and period $j$ by $q_{j}^{i}, p_{j}^{i}, \tilde{h}_{j}^{i}$ and $Q_{j}^{i}$ respectively, where $d_{j}^{i}$ and $Q_{j}^{i}$ are rationals.

We define $x_{j}^{i}$ to be the amount of production of item $i \in I_{0}$ in period $j \in[1, n], s_{j}^{i}$ to be the amount of item $i$ in the inventory at the end of period $j \in[0, n]$, and $y_{j}^{i}$ to be 1 if a setup for item $i$ takes place in period $j \in[1, n]$ and to be 0 otherwise. We can model the two-level multi-item lot-sizing problem $(2 L S)$ as follows.

$$
\begin{array}{cl}
z^{2 L S}=\min _{i \in I_{0}}\left(\tilde{h}_{0}^{i} s_{0}^{i}+\sum_{j=1}^{n}\left(q_{j}^{i} y_{j}^{i}+p_{j}^{i} x_{j}^{i}+\tilde{h}_{j}^{i} s_{j}^{i}\right)\right) & \\
\text { s.t. } s_{j-1}^{0}+x_{j}^{0}=\sum_{i \in I} x_{j}^{i}+s_{j}^{0} & j \in[1, n], \\
s_{j-1}^{i}+x_{j}^{i}=d_{j}^{i}+s_{j}^{i} & i \in I, j \in[1, n], \\
x_{j}^{i} \leq Q_{j}^{i} y_{j}^{i} & i \in I_{0}, j \in[1, n], \\
s_{j}^{i} \geq 0 & i \in I_{0}, j \in[0, n], \\
y_{j}^{i} \in\{0,1\} & i \in I_{0}, j \in[1, n], \\
x_{j}^{i} \geq 0 & i \in I_{0}, j \in[1, n] .
\end{array}
$$

Constraints (2) and (3) are balance constraints for item 0 and items in set $I$, respectively. Constraints (4) relate the production and setup variables and impose the capacity restrictions. Constraints (5)-(7) are variable restrictions. The objective function (1) is the sum of the setup, production and inventory holding costs.

Wagner-Whitin, or non-speculative cost relaxations play an important role in several single level lot-sizing variants. The idea is to obtain a relaxation involving only the $(s, y)$ variables that solves the original lot-sizing problem when the variable costs are such that, given the set-up periods, it is optimal to produce as late as possible. We now derive a relaxation just involving the $(s, y)$ variables for $2 L S$. The approach taken is to first eliminate the production variables from the objective function by substitution, and then relax the constraints by replacing occurrences of the production variables $x_{j}^{i}$ using the variable upper bounds.

In the sequel, we use $a_{u t}$ to denote $\sum_{j=u}^{t} a_{j}$ for both variables and data with $a_{u t}=0$ if $t<u$ and $a^{+}=\max (a, 0)$. 
Substituting $x_{j}^{i}=d_{j}^{i}+s_{j}^{i}-s_{j-1}^{i}$ for $i \in I$ and $x_{j}^{0}=\sum_{i \in I} x_{j}^{i}+s_{j}^{0}-s_{j-1}^{0}=$ $\sum_{i \in I_{0}} s_{j}^{i}+\sum_{i \in I} d_{j}^{i}-\sum_{i \in I_{0}} s_{j-1}^{i}$ for $j \in[1, n]$ in the variable production costs yields

$$
\begin{aligned}
\sum_{i \in I_{0}} \sum_{j=1}^{n} p_{j}^{i} x_{j}^{i}= & \sum_{j=1}^{n}\left(p_{j}^{0}\left(\sum_{i \in I_{0}} s_{j}^{i}+\sum_{i \in I} d_{j}^{i}-\sum_{i \in I_{0}} s_{j-1}^{i}\right)+\sum_{i \in I} p_{j}^{i}\left(d_{j}^{i}+s_{j}^{i}-s_{j-1}^{i}\right)\right) \\
= & \sum_{i \in I} \sum_{j=1}^{n}\left(p_{j}^{0}+p_{j}^{i}\right) d_{j}^{i}+\sum_{j=0}^{n}\left(p_{j}^{0}-p_{j+1}^{0}\right) s_{j}^{0} \\
& +\sum_{i \in I} \sum_{j=0}^{n}\left(p_{j}^{0}-p_{j+1}^{0}+p_{j}^{i}-p_{j+1}^{i}\right) s_{j}^{i},
\end{aligned}
$$

where $p_{0}^{i}=p_{n+1}^{i}=0$ for all $i \in I_{0}$.

For $j \in[0, n]$, let $h_{j}^{0}=p_{j}^{0}-p_{j+1}^{0}+\tilde{h}_{j}^{0}$ and $h_{j}^{i}=p_{j}^{0}-p_{j+1}^{0}+p_{j}^{i}-p_{j+1}^{i}+\tilde{h}_{j}^{i}$ for $i \in I$. Also, let $K=\sum_{i \in I} \sum_{j=1}^{n}\left(p_{j}^{0}+p_{j}^{i}\right) d_{j}^{i}$. Note that the condition $h_{j}^{0} \geq 0$ is the standard non-speculative cost, or Wagner-Whitin condition for the upper level item, and $h_{j}^{i}-h_{j}^{0}=\left(p_{j}^{i}+\tilde{h}_{j}^{i}\right)-\left(\tilde{h}_{j}^{0}+p_{j+1}^{i}\right) \geq 0$ is the similar condition that it is not more expensive to delay transformation/transportation to clients (excluding fixed costs). This is a realistic assumption in many supply chain applications because adding value later in the production process will free capital, and storing end products is usually more costly because of smaller packaging sizes.

Let $1 \leq k \leq t \leq n, l(i) \in[t, n]$ for $i \in I$, and $(x, s, y)$ be a feasible solution to $2 L S$. Summing up (2) for $j \in[k, t]$ and (3) for $j \in[k, l(i)]$ and $i \in I$ gives $\sum_{i \in I_{0}} s_{k-1}^{i}+\sum_{j=k}^{t} x_{j}^{0}+\sum_{i \in I} \sum_{j=t+1}^{l(i)} x_{j}^{i}=\sum_{i \in I} d_{k, l(i)}^{i}+s_{t}^{0}+\sum_{i \in I} s_{l(i)}^{i}$. Since $Q_{j}^{0} y_{j}^{0} \geq x_{j}^{0}$ for $j \in[k, t], Q_{j}^{i} y_{j}^{i} \geq x_{j}^{i}$ for $j \in[t+1, l(i)]$ and $i \in I, s_{t}^{0} \geq 0$, and $s_{l(i)}^{i} \geq 0$ for $i \in I,(x, s, y)$ satisfies

$$
\begin{gathered}
\sum_{i \in I_{0}} s_{k-1}^{i}+\sum_{j=k}^{t} Q_{j}^{0} y_{j}^{0}+\sum_{i \in I} \sum_{j=t+1}^{l(i)} Q_{j}^{i} y_{j}^{i} \geq \sum_{i \in I} d_{k, l(i)}^{i} \\
1 \leq k \leq t \leq n, l(i) \in[t, n] \text { for } i \in I .
\end{gathered}
$$

Similarly, the inequality

$$
s_{k-1}^{i}+\sum_{j=k}^{l} Q_{j}^{i} y_{j}^{i} \geq d_{k l}^{i} \quad i \in I, 1 \leq k \leq l \leq n,
$$

is satisfied by any feasible solution $(x, s, y)$. Hence the problem $2 W W$

$$
z^{2 W W}=K+\min \sum_{i \in I_{0}}\left(h_{0}^{i} s_{0}^{i}+\sum_{j=1}^{n}\left(q_{j}^{i} y_{j}^{i}+h_{j}^{i} s_{j}^{i}\right)\right)
$$

s.t. (5), (6), (8) and (9) 
is a relaxation of $2 L S$. We refer to this relaxation as the two-level Wagner-Whitin relaxation. Next we show that under "non-speculative" cost conditions presented above, this relaxation yields the same optimal value as the original problem.

Proposition 1 If $h_{j}^{i} \geq h_{j}^{0} \geq 0$ for all $i \in I$ and $j \in[0, n]$, then $z^{2 L S}=z^{2 W W}$.

Proof Let $(s, y)$ be an optimal solution to the problem $2 W W$. We will show that if we define the value of $x$ using (2)-(3), the corresponding point $(s, y, x)$ is feasible in $2 L S$ with the same objective function value. As $2 W W$ is a relaxation of $2 L S$, the claim will then follow.

For $i \in I_{0}$, as $h_{n}^{i} \geq 0$, there exists an optimal solution to $2 W W$ with $s_{n}^{i}=0$. For $i \in I$, if there exists $k \in[1, n]$ with $s_{k-1}^{i}>0$ and $s_{k-1}^{i}+\sum_{j=k}^{l} Q_{j}^{i} y_{j}^{i}>d_{k l}^{i}$ for all $l \in[k, n]$, then the solution obtained by decreasing $s_{k-1}^{i}$ and increasing $s_{k-1}^{0}$ by a small amount does not cost more. If there exists $k \in[1, n]$ with $s_{k-1}^{0}>0$ and $\sum_{i \in I_{0}} s_{k-1}^{i}+\sum_{j=k}^{t} Q_{j}^{0} y_{j}^{0}+\sum_{i \in I} \sum_{j=t+1}^{l(i)} Q_{j}^{i} y_{j}^{i}>\sum_{i \in I} d_{k, l(i)}^{i}$ for all choices of $t$ and $l(i)$ for $i \in I$, then the solution obtained by decreasing $s_{k-1}^{0}$ by a small amount is feasible and not worse in terms of cost.

Let $(s, y)$ be an optimal solution to $2 W W$ such that (i) for each $i \in I$ and $k \in[1, n]$ with $s_{k-1}^{i}>0$, there exists $l \in[k, n]$ with $s_{k-1}^{i}+\sum_{j=k}^{l} Q_{j}^{i} y_{j}^{i}=d_{k l}^{i}$, (ii) for each $k \in[1, n]$ with $s_{k-1}^{0}>0$, there exist $t \in[k, n]$ and $l(i) \in[t, n]$ for $i \in I$ with $\sum_{i \in I_{0}} s_{k-1}^{i}+\sum_{j=k}^{t} Q_{j}^{0} y_{j}^{0}+\sum_{i \in I} \sum_{j=t+1}^{l(i)} Q_{j}^{i} y_{j}^{i}=\sum_{i \in I} d_{k, l(i)}^{i}$, and (iii) $s_{n}^{i}=0$ for $i \in I_{0}$.

For $i \in I$ and $k \in[1, n]$, let $x_{k}^{i}=s_{k}^{i}+d_{k}^{i}-s_{k-1}^{i}$. First we show that $x_{k}^{i} \geq 0$. If $s_{k-1}^{i}=0$, then $x_{k}^{i}=s_{k}^{i}+d_{k}^{i} \geq 0$. If $s_{k-1}^{i}>0$, then there exists $l \in[k, n]$ with $s_{k-1}^{i}=d_{k l}^{i}-\sum_{j=k}^{l} Q_{j}^{i} y_{j}^{i}$ and $x_{k}^{i}=s_{k}^{i}+d_{k}^{i}-d_{k l}^{i}+\sum_{j=k}^{l} Q_{j}^{i} y_{j}^{i}=s_{k}^{i}-d_{k+1, l}^{i}+$ $\sum_{j=k+1}^{l} Q_{j}^{i} y_{j}^{i}+Q_{k}^{i} y_{k}^{i}$. Since $s_{k}^{i}+\sum_{j=k+1}^{l} Q_{j}^{i} y_{j}^{i} \geq d_{k+1, l}^{i}$ and $Q_{k}^{i} y_{k}^{i} \geq 0$, we have $x_{k}^{i} \geq 0$. Next we show that $x_{k}^{i} \leq Q_{k}^{i} y_{k}^{i}$. If $s_{k}^{i}=0$, then $x_{k}^{i}=d_{k}^{i}-s_{k-1}^{i} \leq Q_{k}^{i} y_{k}^{i}$. If $s_{k}^{i}>0$, then there exists $l \in[k+1, n]$ with $s_{k}^{i}=d_{k+1, l}^{i}-\sum_{j=k+1}^{l} Q_{j}^{i} y_{j}^{i}$ and $x_{k}^{i}=d_{k+1, l}^{i}-\sum_{j=k+1}^{l} Q_{j}^{i} y_{j}^{i}+d_{k}^{i}-s_{k-1}^{i}=d_{k l}^{i}-\sum_{j=k}^{l} Q_{j}^{i} y_{j}^{i}-s_{k-1}^{i}+Q_{k}^{i} y_{k}^{i}$. As $s_{k-1}^{i}+\sum_{j=k}^{l} Q_{j}^{i} y_{j}^{i} \geq d_{k l}^{i}$, we have $x_{k}^{i} \leq Q_{k}^{i} y_{k}^{i}$.

For $k \in[1, n]$, we take $x_{k}^{0}=\sum_{i \in I} x_{k}^{i}+s_{k}^{0}-s_{k-1}^{0}=\sum_{i \in I_{0}} s_{k}^{i}+\sum_{i \in I} d_{k}^{i}-$ $\sum_{i \in I_{0}} s_{k-1}^{i}$. We first show that $x_{k}^{0} \geq 0$. If $\sum_{i \in I_{0}} s_{k-1}^{i}=0$, then $x_{k}^{0} \geq 0$. Otherwise, if there exist $t \in[k, n]$ and $l(i) \in[t, n]$ for $i \in I$ with $\sum_{i \in I_{0}} s_{k-1}^{i}+\sum_{j=k}^{t} Q_{j}^{0} y_{j}^{0}+$ $\sum_{i \in I} \sum_{j=t+1}^{l(i)} Q_{j}^{i} y_{j}^{i}=\sum_{i \in I} d_{k, l(i)}^{i}$, then

$$
\begin{aligned}
x_{k}^{0} & =\sum_{i \in I_{0}} s_{k}^{i}+\sum_{i \in I} d_{k}^{i}+\sum_{j=k}^{t} Q_{j}^{0} y_{j}^{0}+\sum_{i \in I} \sum_{j=t+1}^{l(i)} Q_{j}^{i} y_{j}^{i}-\sum_{i \in I} d_{k, l(i)}^{i} \\
& =Q_{k}^{0} y_{k}^{0}+\sum_{i \in I_{0}} s_{k}^{i}+\sum_{j=k+1}^{t} Q_{j}^{0} y_{j}^{0}+\sum_{i \in I} \sum_{j=t+1}^{l(i)} Q_{j}^{i} y_{j}^{i}-\sum_{i \in I} d_{k+1, l(i)}^{i} \geq 0 .
\end{aligned}
$$


If no such $t$ and $l(i)$ for $i \in I$ exist, then $s_{k-1}^{0}=0$. Let $I^{\prime}=\left\{i \in I: s_{k-1}^{i}>0\right\}$. For each $i \in I^{\prime}$, there exists $l(i) \in[k, n]$ with $s_{k-1}^{i}=d_{k, l(i)}^{i}-\sum_{j=k}^{l(i)} Q_{j}^{i} y_{j}^{i}$, and

$$
\begin{aligned}
x_{k}^{0} & =\sum_{i \in I_{0}} s_{k}^{i}+\sum_{i \in I} d_{k}^{i}-\sum_{i \in I^{\prime}}\left(d_{k, l(i)}^{i}-\sum_{j=k}^{l(i)} Q_{j}^{i} y_{j}^{i}\right) \\
& =\sum_{i \in I_{0} \backslash I^{\prime}} s_{k}^{i}+\sum_{i \in I \backslash I^{\prime}} d_{k}^{i}+\sum_{i \in I^{\prime}} Q_{k}^{i} y_{k}^{i}+\sum_{i \in I^{\prime}}\left(s_{k}^{i}-d_{k+1, l(i)}^{i}+\sum_{j=k+1}^{l(i)} Q_{j}^{i} y_{j}^{i}\right) \geq 0 .
\end{aligned}
$$

Now we show that $x_{k}^{0} \leq Q_{k}^{0} y_{k}^{0}$. If $\sum_{i \in I_{0}} s_{k}^{i}=0$, then $x_{k}^{0}=\sum_{i \in I} d_{k}^{i}-\sum_{i \in I_{0}} s_{k-1}^{i} \leq$ $Q_{k}^{0} y_{k}^{0}$ (inequality (8) for $t=k$ and $l(i)=k$ for all $i \in I$ ). Otherwise, if there exist $t \in[k+1, n]$ and $l(i) \in[t, n]$ with $\sum_{i \in I_{0}} s_{k}^{i}+\sum_{j=k+1}^{t} Q_{j}^{0} y_{j}^{0}+\sum_{i \in I} \sum_{j=t+1}^{l(i)} Q_{j}^{i} y_{j}^{i}=$ $\sum_{i \in I} d_{k+1, l(i)}^{i}$, then

$$
\begin{aligned}
x_{k}^{0} & =\sum_{i \in I} d_{k+1, l(i)}^{i}-\sum_{j=k+1}^{t} Q_{j}^{0} y_{j}^{0}-\sum_{i \in I} \sum_{j=t+1}^{l(i)} Q_{j}^{i} y_{j}^{i}+\sum_{i \in I} d_{k}^{i}-\sum_{i \in I_{0}} s_{k-1}^{i} \\
& =\sum_{i \in I} d_{k, l(i)}^{i}+Q_{k}^{0} y_{k}^{0}-\sum_{j=k}^{t} Q_{j}^{0} y_{j}^{0}-\sum_{i \in I} \sum_{j=t+1}^{l(i)} Q_{j}^{i} y_{j}^{i}-\sum_{i \in I_{0}} s_{k-1}^{i} \leq Q_{k}^{0} y_{k}^{0} .
\end{aligned}
$$

If no such $t$ and $l(i)$ for $i \in I$ exist, then $s_{k}^{0}=0$. Let $I^{\prime}=\left\{i \in I: s_{k}^{i}>0\right\}$. For each $i \in I^{\prime}$, there exists $l(i) \in[k+1, n]$ with $s_{k}^{i}=d_{k+1, l(i)}^{i}-\sum_{j=k+1}^{l(i)} Q_{j}^{i} y_{j}^{i}$. In this case,

$$
\begin{aligned}
x_{k}^{0} & =\sum_{i \in I^{\prime}}\left(d_{k+1, l(i)}^{i}-\sum_{j=k+1}^{l(i)} Q_{j}^{i} y_{j}^{i}\right)+\sum_{i \in I} d_{k}^{i}-\sum_{i \in I_{0}} s_{k-1}^{i} \\
& =Q_{k}^{0} y_{k}^{0}+\sum_{i \in I^{\prime}}\left(d_{k, l(i)}^{i}-\sum_{j=k+1}^{l(i)} Q_{j}^{i} y_{j}^{i}\right)+\sum_{i \in I \backslash I^{\prime}} d_{k}^{i}-\sum_{i \in I_{0}} s_{k-1}^{i}-Q_{k}^{0} y_{k}^{0} \leq Q_{k}^{0} y_{k}^{0},
\end{aligned}
$$

since $\sum_{i \in I^{\prime}}\left(d_{k, l(i)}^{i}-\sum_{j=k+1}^{l(i)} Q_{j}^{i} y_{j}^{i}\right)+\sum_{i \in I \backslash I^{\prime}} d_{k}^{i}-\sum_{i \in I_{0}} s_{k-1}^{i}-Q_{k}^{0} y_{k}^{0} \leq 0$ by inequality (8) with $t=k$ and $l(i)=k$ for all $i \in I \backslash I^{\prime}$.

Now as $0 \leq x_{k}^{i} \leq Q_{k}^{i} y_{k}^{i}$ for all $i \in I_{0}$ and $k \in[1, n]$, the solution $(x, s, y)$ is feasible for $2 L S$.

Defining $X^{2 W W}$ as the set of solutions to (8)-(9) and the associated bound and integrality constraints (5)-(6) and $\bar{X}_{k}^{2 D L S}$, for fixed $k \in[1, n]$, as

$$
\begin{aligned}
& \sum_{i \in I_{0}} s_{k-1}^{i}+\sum_{j=k}^{t} Q_{j}^{0} y_{j}^{0}+\sum_{i \in I} \sum_{j=t+1}^{l(i)} Q_{j}^{i} y_{j}^{i} \geq \sum_{i \in I} d_{k, l(i)}^{i} \\
& k \leq t \leq n, l(i) \in[t, n] \quad \text { for } i \in I,
\end{aligned}
$$




$$
\begin{aligned}
& s_{k-1}^{i}+\sum_{j=k}^{l} Q_{j}^{i} y_{j}^{i} \geq d_{k l}^{i} \quad i \in I, l \in[k, n], \\
& s_{k-1}^{i} \in \mathbb{R}_{+}^{1}, y_{j}^{i} \in\{0,1\} \quad i \in I_{0}, j \in[k, n],
\end{aligned}
$$

it is easy to see that $X^{2 W W}=\bigcap_{k=1}^{n+1} \bar{X}_{k}^{2 D L S}$. Moreover each of the sets $\bar{X}_{k}^{2 D L S}$ is of the same form. It is natural to hope that with a good approximation or an exact formulation for conv $\left(\bar{X}_{k}^{2 D L S}\right)$, the intersection of these formulations will provide a good approximation of $\operatorname{conv}\left(X^{2 W W}\right)$.

However, in the next section, we will analyze a slightly different set for the following reason. We remark that $X^{2 W W}$ may have extreme points that are not feasible for $2 L S$. Because of the cost conditions $h_{k}^{0} \leq h_{k}^{i}$ for all $i \in I$ and $k \in[0, n-1]$, these extreme points will not be unique optimal solutions. The same is true for $\bar{X}_{k}^{2 D L S}$. Consider then the set $X_{k}^{2 D L S}$ defined similarly to $\bar{X}_{k}^{2 D L S}$, except that we generate inequalities of the form (10) for all subsets of items $V \subseteq I$ as

$$
\begin{gathered}
\sum_{i \in V \cup\{0\}} s_{k-1}^{i}+\sum_{j=k}^{t} Q_{j}^{0} y_{j}^{0}+\sum_{i \in V} \sum_{j=t+1}^{l(i)} Q_{j}^{i} y_{j}^{i} \geq \sum_{i \in V} d_{k, l(i)}^{i} \\
\emptyset \subset V \subseteq I, t \in[k-1, n], l(i) \in[t, n] \quad \text { for } i \in V .
\end{gathered}
$$

The idea is that if $h_{k-1}^{0}>h_{k-1}^{i}$, decreasing $s_{k-1}^{0}$ and increasing $s_{k-1}^{i}$ by $s_{k-1}^{0}$ improves the objective function value without violating (10)-(12). But this new solution will be infeasible in $2 L S$ if the inventory $s_{k-1}^{0}$ is used in the solution to satisfy demand for some item other than $i$. Constraints (13) forbid this type of solution.

Note that minimizing the objective function $\sum_{i \in I_{0}}\left(h_{0}^{i} s_{0}^{i}+\sum_{j=1}^{n} f_{j}^{i} y_{j}^{i}\right)$ over $X_{1}^{2 D L S}$ solves $2 L S$ when $p_{j}^{i}=0$ for all $j \in[1, n], h_{0}^{i} \geq 0$ and $h_{j}^{i}=0$ for all $j \in[1, n]$ and $i \in I_{0}$. We call this problem the two-level discrete lot-sizing problem (2DLS). In the case of $2 D L S$ we do not need the conditions $h_{0}^{0} \leq h_{0}^{i}$ for all $i \in I$ to have a valid formulation for $2 D L S$, because of the strengthened constraints (13). It is worth noting that this is not true for $2 W W$ : Proposition 1 does not hold if the assumption that $h_{k}^{0} \leq h_{k}^{i}$ for all $i \in I$ and $k \in[0, n-1]$ is dropped, even when one replaces constraints (10) by constraints (13) for all $k$.

\section{The two-level discrete lot-sizing problem $2 D L S$}

In this section, we consider the structure of $X^{2 D L S}=X_{1}^{2 D L S}$ when $Q_{j}^{0}=M$ is large $\left(M \geq \sum_{i \in I} d_{1 n}^{i}\right)$ for all $j \in[1, n]$ except in Sect. 3.4. Let $e_{\alpha}$ denote the $\alpha$ th unit vector and $e_{0}$ or $e_{n+1}$ the 0 -vector in $\mathbb{R}^{n}$.

Observation 1 Every extreme point of $\operatorname{conv}\left(X^{2 D L S}\right)$ has $y^{0}=e_{\alpha}$ for some $\alpha \in$ $\{1, \ldots, n+1\}$.

The following result allows us to largely decompose the problem by item. Let $\phi^{i}$ denote the contribution (if any) of item $i \in I$ to the upper level stock $s_{0}^{0}$. 


\section{Proposition 2}

$$
\begin{aligned}
& s_{0}^{0}=\sum_{i \in I} \phi^{i}, \\
& \phi^{i}+s_{0}^{i}+M y_{1 t}^{0}+\sum_{j=t+1}^{l} Q_{j}^{i} y_{j}^{i} \geq d_{1 l}^{i} \quad i \in I, l \in[1, n], t \in[0, l], \\
& s_{0}^{i}+\sum_{j=1}^{l} Q_{j}^{i} y_{j}^{i} \geq d_{1 l}^{i} \quad i \in I, l \in[1, n] \\
& s_{0} \in \mathbb{R}_{+}^{m+1}, y \in\{0,1\}^{(m+1) n}, \phi \in \mathbb{R}_{+}^{m} .
\end{aligned}
$$

is an extended formulation for $X^{2 D L S}$.

Proof Suppose that $\left(s_{0}, y, \phi\right)$ satisfies (15)-(17). Let $V \subseteq I, k=1, t \in[0, n]$ and $l(i) \in[t, n]$ for $i \in V$. Summing (15) for $l=l(i)$ over $i \in V$ yields $\sum_{i \in V} \phi^{i}+$ $\sum_{i \in V} s_{0}^{i}+|V| M y_{1 t}^{0}+\sum_{i \in V} \sum_{j=t+1}^{l} Q_{j}^{i} y_{j}^{i} \geq \sum_{i \in V} d_{1 l(i)}^{i}$. As $s_{0}^{0} \geq \sum_{i \in V} \phi^{i}, M \geq$ $\sum_{i \in V} d_{1 l(i)}^{i}$ and $y$ is binary, $\left(s_{0}, y\right)$ satisfies (13). Hence we can conclude that $\left(s_{0}, y\right)$ is in $X^{2 D L S}$.

Let $\left(s_{0}, y\right)$ be an extreme point of $\operatorname{conv}\left(X^{2 D L S}\right)$ with $y^{0}=e_{\alpha}$. Then we know that $s_{0}^{0}=\sum_{i \in I} \max _{l \in[\alpha-1, n]}\left(d_{1 l}^{i}-\sum_{j=\alpha}^{l} Q_{j}^{i} y_{j}^{i}-s_{0}^{i}\right)^{+}$. We can verify that $\left(s_{0}, y, \phi\right)$ with $\phi^{i}=\max _{l \in[\alpha-1, n]}\left(d_{1 l}^{i}-\sum_{j=\alpha}^{l} Q_{j}^{i} y_{j}^{i}-s_{0}^{i}\right)^{+}$for $i \in I$ satisfies (15)-(17).

\subsection{Uncapacitated at both levels $2 D L S-(U, U)$}

Now we suppose that $Q_{j}^{i}=M$ for all $i \in I_{0}$ and $j \in[1, n]$ and we replace the constraints $y^{i} \in\{0,1\}^{n}$ by $y^{i} \in \mathbb{Z}_{+}^{n}$ for all $i \in I_{0}$.

Observation 2 Every extreme point of $\operatorname{conv}\left(X^{2 D L S-(U, U)}\right)$ has $y^{0}=e_{\alpha}$ for some $\alpha \in[1, n+1]$ and $y_{i}^{1}=e_{\beta_{i}}$ or $y^{1}=e_{\beta_{i}}+e_{\gamma_{i}}$ where $\beta_{i} \in[\alpha, n+1]$ and $\gamma_{i} \in[0, \alpha-1]$.

This observation directly leads to a $\mathcal{O}\left(n^{3} m\right)$ combinatorial algorithm for solving $2 D L S-(U, U)$. Note that the problem is a special case of the NP-Hard One-WarehouseMultiple-Retailer problem (OWMR) [3], where the variable production costs and the holding costs (except for the initial inventories) are zero.

The constraints (15) now take the form

$$
\phi^{i}+s_{0}^{i}+M y_{1 t}^{0}+M y_{t+1, l}^{i} \geq d_{1 l}^{i}
$$

We see that the demand $d_{l}^{i}$ must be satisfied from the initial stock term $\phi^{i}+s_{0}^{i}$ if $y_{1 t}^{0}+y_{t+1, l}^{i}=0$ for some $t \in[0, l]$. Taking $\zeta_{l}^{i}$ to represent $\max _{t \in[0, l]}\left(1-y_{1 t}^{0}-y_{t+1, l}^{i}\right)^{+}$ and $\delta_{l}^{i}$ to represent $\left(1-y_{1 l}^{i}\right)^{+}$, one obtains the extended formulation: 


$$
\begin{array}{ll}
s_{0}^{0}=\sum_{i \in I} \phi^{i}, & \\
\phi^{i}+s_{0}^{i}=\sum_{l=1}^{n} d_{l}^{i} \zeta_{l}^{i} & i \in I, \\
s_{0}^{i}=\sum_{l=1}^{n} d_{l}^{i} \delta_{l}^{i} & i \in I, \\
\zeta_{l}^{i} \geq \delta_{l}^{i} & i \in I, l \in[1, n], \\
\zeta_{l}^{i}+y_{1 t}^{0}+y_{t+1, l}^{i} \geq 1 & i \in I, l \in[1, n], t \in[0, l], \\
\delta_{l}^{i}+y_{1 l}^{i} \geq 1 & i \in I, l \in[1, n], \\
\zeta \in \mathbb{R}_{+}^{m n}, \delta \in \mathbb{R}_{+}^{m n}, y \in \mathbb{R}_{+}^{(m+1) n}, & \\
\zeta \in \mathbb{Z}^{m n}, \delta \in \mathbb{Z}^{m n}, y \in \mathbb{Z}^{(m+1) n} . &
\end{array}
$$

Note that the constraints (21) are necessary to obtain a correct formulation when conditions $h_{0}^{0} \leq h_{0}^{i}$ for all $i \in I$ are not satisfied. Let $S C$ be the set-covering polyhedron described by the constraints (22)-(24) and $S C^{\prime}$ be $S C \cap$ (21).

Theorem 1 The polyhedron $S C^{\prime}$ is integral.

The proof is in three steps. First we will establish the result for the polyhedron $S C$ when $m=1$. We then extend this result for all values of $m$. Finally we show that adding constraints (21) does not create fractional extreme points. Note that the $0-1$ constraint matrix associated to $S C$ is neither totally unimodular (TU) nor balanced.

Theorem 2 The polyhedron SC is integral when $m=1$.

Proof We drop the index $i$ in $\zeta_{l}^{i}$ and $\delta_{l}^{i}$ as $m=1$. To show integrality we adopt the approach of Lovasz [16]. Given a non-zero objective function $(g, q)$, let $M(g, q)$ denote the set of optimal solutions to the integer program: $\min \left\{\sum_{u=1}^{n} g_{u}^{0} \zeta_{u}+\right.$ $\sum_{u=1}^{n} g_{u}^{1} \delta_{u}+\sum_{i=0}^{1} \sum_{u=1}^{n} q_{u}^{i} y_{u}^{i}:(\zeta, \delta, y)$ satisfy (22) - (25) $\}$. We will show that when the optimal value is finite, $M(g, q) \subset\{x: a x=b\}$ where $a x \geq b$ is one of the constraints (22)-(24).

The extreme rays $\left(y^{0}, y^{1}, \zeta, \delta\right)$ of $S C$ are $\left(e_{j}, 0,0,0\right),\left(0, e_{j}, 0,0\right),\left(0,0, e_{j}, 0\right)$ and $\left(0,0,0, e_{j}\right)$ for $j \in[1, n]$. Hence we need $g^{0}, g^{1}, q^{0}, q^{1} \geq 0$ for the objective value to be bounded.

If $g^{0}=g^{1}=0$, then there exists $i, u$ with $q_{u}^{i}>0$ and all optimal solutions satisfy $y_{u}^{i}=0$. If $q_{u}^{0}<q_{u+1}^{0}$ for some $u$, then $y_{u+1}^{0}=0$. Therefore, for the remaining cases, we assume that there exists $t \in[0, n]$ such that $q_{1}^{0} \geq q_{2}^{0} \geq \cdots \geq q_{t}^{0}>0=q_{t+1}^{0}=$ $\cdots=q_{n}^{0}$. If $g^{0}=0$ and there exists $u$ with $q_{u}^{0}>0$, then $y_{u}^{0}=0$. If $g^{0}=q^{0}=0$, then the problem is single-level and the result is known to hold [21].

In the remaining case, there exists $l$ such that $g_{l}^{0}>0$. Let $l$ be the highest such index. If there exists $k \in[1, l]$ such that $q_{k}^{0}+q_{k}^{1}<g_{l}^{0}$ then $\zeta_{l}=0$. If $t>l$, then $y_{t}^{0}=0$. Suppose that $t \leq l$ and $q_{k}^{1}=q_{k}^{0}+q_{k}^{1} \geq g_{l}^{0}>0$ for $t<k \leq l$. We claim that all optimal solutions satisfy inequality (22) at equality for this choice of $t$ and $l$. 
Observe that all variables in the inequality have positive cost, and hence showing the result for all optimal extreme points of the convex hull of solutions of (22)-(25) is sufficient.

By Observation 2 an extreme point is of the form $y^{0}=e_{\alpha}$, and $y^{1}=e_{\beta}$ or $y^{1}=e_{\beta}+e_{\gamma}$ where $\beta \geq \alpha$ and $\gamma<\alpha$. Let $\left(y^{0}, y^{1}, \zeta, \delta\right)$ be an extreme point. We look at three cases.

1. $\zeta_{l}=1$. Then $\beta>l$. If $\alpha \leq t$, then setting $y^{0}=e_{t+1}$ improves the cost by $q_{\alpha}^{0}>0$. If $t+1 \leq \gamma \leq l$, then setting $y^{0}=y^{1}=e_{\gamma}$ and $\zeta_{l}=0$ improves the cost by at least $g_{l}^{0}>0$. Otherwise $(\alpha>t$ and $\gamma<t+1$ or $\gamma>l)$ the inequality (22) is satisfied at equality.

2. $\zeta_{l}=0$ and $\alpha \leq t$. If $t+1 \leq \beta$, then setting $y^{0}=e_{t+1}$ improves the cost by $q_{\alpha}^{0}>0$. Otherwise, the claim holds.

3. $\zeta_{l}=0$ and $\alpha \geq t+1$. If $t+1 \leq \gamma<\alpha \leq \beta \leq l$, then setting $y^{0}=y^{1}=e_{\gamma}$ improves the cost by $q_{\beta}^{1}>0$. Otherwise, the claim holds.

To extend the result to cover multiple items, we first present a somewhat abstract proposition that will then be applied to the set covering problem.

For $k=1, \ldots, K$, consider the polyhedron $P^{k}$

$$
\begin{array}{r}
A w^{0}+B w^{c} \geq \mathbf{1} c=1, \ldots, k \\
w^{0} \in \mathbb{R}_{+}^{n}, w^{c} \in \mathbb{R}_{+}^{n_{1}} \quad c=1, \ldots, k,
\end{array}
$$

where $A, B \geq 0$ are rational matrices and $X^{k}=P^{k} \cap \mathbb{Z}^{N^{k}}$ with $N^{k}=n+k n_{1}$. Suppose that

i. For all $k$ and in every extreme point of $\operatorname{conv}\left(X^{k}\right), \sum_{j=1}^{n} w_{j}^{0} \leq 1$,

ii. for every $\left(w^{0}, w^{1}\right) \in P^{1}$ with $\sum_{j=1}^{n} w_{j}^{0}>1$, there exists a point $\left(\bar{w}^{0}, w^{1}\right) \in P^{1}$ such that $\bar{w}^{0} \leq w^{0}, \sum_{j=1}^{n} \bar{w}_{j}^{0}=1$ and $\min \left(\mathbf{1}, A w^{0}\right)=\min \left(\mathbf{1}, A \bar{w}^{0}\right)$ componentwise,

iii. $P^{1}$ is an integral polyhedron,

iv. $W^{\alpha}=\left\{\left(w^{0}, w^{1}\right) \in \mathbb{R}_{+}^{n} \times \mathbb{R}_{+}^{n_{1}}: w^{0}=e_{\alpha}, B w^{1} \geq \mathbf{1}-A e_{\alpha}\right\}$ is an integral polyhedron for all $\alpha \in[1, n+1]$.

Proposition 3 Under the above conditions, $P^{k}$ is an integral polyhedron for all $k \geq 1$.

Proof First we observe that from (i),

$$
X^{k}=\cup_{\alpha=1}^{n+1}\left(X^{k} \cap\left\{w: w^{0}=e_{\alpha}\right\}\right)+\mathbb{Z}_{+}^{N^{k}} .
$$

From (iii)

$$
P^{1}=\operatorname{conv}\left(X^{1}\right)=\operatorname{conv}\left(\cup_{\alpha=1}^{n+1} \operatorname{conv}\left(X^{1} \cap\left\{w: w^{0}=e_{\alpha}\right\}\right)\right)+\mathbb{R}_{+}^{N^{1}}
$$

and for $k>1$ we have

$$
\operatorname{conv}\left(X^{k}\right)=\operatorname{conv}\left(\cup_{\alpha=1}^{n+1} \operatorname{conv}\left(X^{k} \cap\left\{w: w^{0}=e_{\alpha}\right\}\right)\right)+\mathbb{R}_{+}^{N^{k}} \subseteq P^{k} .
$$




\section{By (iv)}

$$
\operatorname{conv}\left(X^{1} \cap\left\{w: w^{0}=e_{\alpha}\right\}\right)=\left\{\left(w^{0}, w^{1}\right): w^{0}=e_{\alpha}, B w^{1} \geq \mathbf{1}-A e_{\alpha}, w^{1} \in \mathbb{R}_{+}^{n_{1}}\right\} .
$$

Now consider a point $\left(w^{0}, w^{1}\right) \in P^{1}$. If $\sum_{j=1}^{n} w_{j}^{0}>1$, by $(i i)$, there exists a vector $\bar{w}^{0} \in \mathbb{R}_{+}^{n}$ with $\bar{w}^{0} \leq w^{0},\left(\bar{w}^{0}, w^{1}\right) \in P^{1}, \sum_{j=1}^{n} \bar{w}_{j}^{0}=1$ and $\min \left(\mathbf{1}, A w^{0}\right)=$ $\min \left(\mathbf{1}, A \bar{w}^{0}\right)$. Otherwise set $\bar{w}^{0}=w^{0}$.

Now from the representation of $P^{1}$ as the convex hull of the union of polyhedra, we have that there exist $\lambda \in \mathbb{R}_{+}^{n+1}$ with $\sum_{\alpha=1}^{n+1} \lambda_{\alpha}=1$ and points $w^{1, \alpha} \in W^{\alpha}$ for $\alpha=1, \ldots, n+1$ such that

$$
\left(\bar{w}^{0}, w^{1}\right)=\sum_{\alpha=1}^{n+1} \lambda_{\alpha}\left(e_{\alpha}, w^{1, \alpha}\right)
$$

with $\bar{w}_{\alpha}^{0}=\lambda_{\alpha}$ for $\alpha=1, \ldots, n$.

Now consider a point $\left(w^{0}, w^{1}, \ldots, w^{k}\right) \in P^{k}$ and select $\bar{w}^{0}$ as above. Because of the min condition (ii), $\left(\bar{w}^{0}, w^{1}, \ldots, w^{k}\right) \in P^{k}$. For each $c=1, \ldots, k$, the above argument provides points $w^{c, \alpha}$ and weights $\lambda_{\alpha}^{c}$ such that

$$
\left(\bar{w}^{0}, w^{c}\right)=\sum_{\alpha=1}^{n+1} \lambda_{\alpha}^{c}\left(e_{\alpha}, w^{c, \alpha}\right)
$$

Note that $\lambda_{\alpha}^{c}=\lambda_{\alpha}=\bar{w}_{\alpha}^{0}$ for $\alpha=1, \ldots, n$, i.e., the weights are identical for each $c=1, \ldots, k$. Now

$$
\left(w^{0}, w^{1}, \ldots, w^{k}\right) \geq\left(\bar{w}^{0}, w^{1}, \ldots, w^{k}\right)=\sum_{\alpha=1}^{n+1} \lambda_{\alpha}\left(e_{\alpha}, w^{1, \alpha}, \ldots, w^{k, \alpha}\right)
$$

Thus we have shown that $P^{k} \subseteq \operatorname{conv}\left(\cup_{\alpha=1}^{n+1} \operatorname{conv}\left(X^{k} \cap\left\{w: w^{0}=e_{\alpha}\right\}\right)\right)+\mathbb{R}_{+}^{N^{k}}$ and thus $P^{k}=\operatorname{conv}\left(X^{k}\right)$.

Proof of Theorem 1 We first apply the above to the polyhedron $S C$ and its associated set $S C_{I}$ of integer points.

To demonstrate that $S C$ is integral, we need to check the four conditions of Proposition 3. Here we have $n_{1}=n$ and we take $w^{0}=y^{0}$.

i. Every extreme point of $\operatorname{conv}\left(S C_{I}\right)$ satisfies $y^{0}=e_{\alpha}$ for some $\alpha \in\{1, \ldots, n+1\}$.

ii. Given $\left(y^{0}, y^{1}, \zeta, \delta\right) \in P^{1}$ with $\sum_{j=1}^{n} y_{j}^{0}>1$, we select $\bar{w}^{0}$ as follows: $\bar{w}^{0}$ is lexicographically maximum subject to $0 \leq \bar{w}^{0} \leq y^{0}$ and $\sum_{j=1}^{n} \bar{w}_{j}^{0}=1$. It is easily verified that $\left(\bar{w}^{0}, y^{1}, \zeta, \delta\right) \in P^{1}$.

iii. (22)-(24) is an integral polyhedron for $m=1$ by Theorem 2 .

iv. $W^{\alpha}$ is the polyhedron obtained by setting $y_{\alpha}^{0}=1$. After eliminating certain unnecessary constraints one obtains for each fixed $i \in I$ : 


$$
\begin{array}{ll}
y^{0}=e_{\alpha}, & \\
\zeta_{l}^{i} \geq 1 & l \in[1, \alpha-1], \\
\zeta_{l}^{i}+y_{\alpha, l}^{i} \geq 1 & l \in[\alpha, n], \\
\delta_{l}^{i}+y_{1, l}^{i} \geq 1 & l \in[1, n], \\
\delta^{i}, \zeta^{i}, y^{i} \in \mathbb{R}_{+}^{n} . &
\end{array}
$$

We will prove that the constraint matrix associated to (28)-(29) is TU. A matrix $B$ is TU if and only if each subset $J$ of its columns can be partitioned into two sets $J_{1}$ and $J_{2}$ such that for each row $r$ we have $\sum_{k \in J_{1}} b_{r k}-\sum_{k \in J_{2}} b_{r k} \in\{0,1,-1\}$ [11]. Given a subset of columns $J$, we put the column associated with the $y_{j}^{i}$ variable with the smallest index $j$ into $J_{1}$, the next one into $J_{2}$, the next into $J_{1}$ and so on. Finally we set $\zeta_{l}^{i}$ and $\delta_{l}^{i}$ in the opposite set to $y_{k}^{i}$ with $k$ the highest index in $J$ smaller than or equal to $l$ (and $J_{1}$ otherwise). It is easily checked that this partition satisfies the property.

Now the integrality of $S C$ follows from Proposition 3.

It remains to show that adding constraints (21) does not create fractional extreme points. For any $J \subseteq I \times[1, n]$, consider the face of $S C^{\prime}$ where (21) is tight for $(i, l) \in J$ and not necessarily tight for $(i, j) \in \bar{J}$. Since any extreme point of $S C^{\prime}$ is also an extreme point of such a face for some $J$, showing that this face is integral for any $J$ implies that $S C^{\prime}$ is integral.

For $(i, l) \in J$, both (23) (dominated by (22) when $t=0$ ) and $\delta_{l}^{i} \geq 0$ can be dropped from the formulation. Then the face reduces to

$$
\begin{array}{ll}
\zeta_{l}^{i}=\delta_{l}^{i} & (i, l) \in J, \\
\zeta_{l}^{i}+y_{1 t}^{0}+y_{t+1, l}^{i} \geq 1 & i \in I, l \in[1, n], t \in[0, l], \\
\delta_{l}^{i}+y_{1 l}^{i} \geq 1 & (i, l) \in \bar{J}, \\
\zeta \in \mathbb{R}_{+}^{m n}, \delta \in \mathbb{R}_{+}^{|\bar{J}|}, y \in \mathbb{R}_{+}^{(m+1) n}, &
\end{array}
$$

It is easy to see that (32)-(34) is the projection of $S C$ with $\delta_{l}^{i}$ for $(i, l) \in J$ being the variables projected out. But this last polyhedron has just been proved to be integral.

We now return to the two-level discrete lot-sizing problem:

$$
\min \left\{\sum_{i \in I_{0}}\left(h_{0}^{i} s_{0}^{i}+\sum_{j=1}^{n} f_{j}^{i} y_{j}^{i}\right) \mid\left(s_{0}, y\right) \in X^{2 D L S-(U, U)}\right\} .
$$

We have shown that it can be solved as a linear program using the extended formulation

$$
\min \left\{\sum_{i \in I_{0}}\left(h_{0}^{i} s_{0}^{i}+\sum_{j=1}^{n} f_{j}^{i} y_{j}^{i}\right) \mid\left(s_{0}, \phi, y, \zeta, \delta\right) \text { satisfying (18) - (24) }\right\}
$$

with $\Theta(m n)$ variables and $\Theta\left(m n^{2}\right)$ constraints. 
Observation 3 Because $\zeta_{l}^{i}=\max _{t \in[0, l]}\left(1-y_{1 t}^{0}-y_{t+1, l}^{i}\right)^{+}$can be rewritten as $\zeta_{l}^{i}=$ $\max \left(\zeta_{l-1}^{i}-y_{l}^{i}, 1-y_{1 l}^{0}\right)^{+}$, a more compact linear program with $\Theta(m n)$ constraints is obtained using the constraints

$$
\begin{aligned}
& \zeta_{0}^{i}=1, \\
& \zeta_{l}^{i} \geq 1-y_{1 l}^{0} \quad i \in I, l \in[1, n], \\
& \zeta_{l}^{i} \geq \zeta_{l-1}^{i}-y_{l}^{i} \quad i \in I, l \in[1, n]
\end{aligned}
$$

in place of (22).

One can also describe the convex hull in the space of the original $\left(s_{0}, y\right)$ variables. By projection, we obtain

Proposition $4 \operatorname{conv}\left(X^{2 D L S-(U, U)}\right)$ is given by:

$$
\begin{aligned}
& s_{0}^{0}+\sum_{i \in V} s_{0}^{i} \geq \sum_{i \in V} \sum_{u=1}^{l(i)} d_{u}^{i}\left(1-y_{1 t(i, u)}^{0}-y_{t(i, u)+1, u}^{i}\right) \\
& V \subseteq I, l(i) \in[1, n], t(i, u) \in\{t(i, u-1), u\}, t(i, 0)=0, \text { for } u \in[1, l(i)] \text { and } i \in V \\
& s_{0}^{i} \geq \sum_{u=1}^{l} d_{u}^{i}\left(1-y_{1 l}^{i}\right) \quad i \in I, l \in[1, n], \\
& s_{0}^{i} \in \mathbb{R}_{+}^{1}, y_{j}^{i} \in \mathbb{R}_{+}^{1} \quad i \in I_{0}, j \in[1, n] .
\end{aligned}
$$

Proof [sketch]: Variables $\phi^{i}$ can first be eliminated by substitution. Then we project variables $\zeta_{l}^{i}$ and obtain inequalities of the form

$$
s_{0}^{0}+\sum_{i \in I} s_{0}^{i} \geq \sum_{l=1}^{n} d_{l}^{i} \underline{\zeta}_{l}^{i}
$$

where each $\underline{\zeta}_{l}^{i}$ represents one of the lower bounds in $(\delta, y)$ derived from (21), (22) or nonnegativity of $\zeta_{l}^{i}$. Projecting out variables $\delta_{l}^{i}$ then similarly amounts to replacing each occurrence of $\delta_{l}^{i}$ by one of the lower bounds in $y$ derived from (23) or nonnegativity. One finally obtains a large class of valid inequalities that includes (38)-(40). Finally, using Observation 3, it is easy to see that (38)-(40) dominates all the other valid inequalities in the class.

Finally observe that the reformulation (35)-(37) of Observation 3 leads to an $\Theta(\mathrm{nm})$ separation algorithm for the inequalities (38). Given $\left(\bar{s}_{0}, \bar{y}\right)$, one calculates

$$
\bar{\zeta}_{l}^{i}=\max \left(1-\bar{y}_{1 l}^{0}, \bar{\zeta}_{l-1}^{i}-\bar{y}_{l}^{i}\right)^{+} \text {and } \bar{\phi}^{i}=\left(\sum_{u=1}^{n} d_{u}^{i} \bar{\zeta}_{u}^{i}-\bar{s}_{0}^{i}\right)
$$

Let $V=\left\{i: \bar{\phi}^{i}>0\right\}$. Then one obtains violated inequality if and only if $\bar{s}_{0}^{0}<\sum_{i \in V} \bar{\phi}^{i}$. 


\subsection{Start-up costs $2 D L S-(U, U)-S C$}

Here we consider the two-level uncapacitated lot-sizing problem with start-ups at both levels. A start-up occurs in the first period of an interval of set-ups. Start-ups often arise at the lower level in make-pack problems. To represent start-ups, we introduce the variables $z_{j}^{i}=1$ if $y_{j}^{i}=1$ and $y_{j-1}^{i}=0$, and $z_{j}^{i}=0$ otherwise. Thus the constraints

$$
\begin{aligned}
z_{j}^{i} & \geq y_{j}^{i}-y_{j-1}^{i} & & i \in I_{0}, j \in[1, n], \\
z_{j}^{i} & \leq y_{j}^{i} & & i \in I_{0}, j \in[1, n], \\
z_{j}^{i} & \in \mathbb{R}_{+} & & i \in I_{0}, j \in[1, n], \\
y_{0}^{i} & \in \mathbb{Z}_{+} & & i \in I_{0},
\end{aligned}
$$

are added to the original formulation $2 L S$.

Specifically we consider the discrete lot-sizing set $X^{2 D L S-(U, U)-S C}$ that is the intersection of $X^{2 D L S-(U, U)}$ and the additional constraints. Following a similar proof in three steps, see "Appendix", one obtains a result similar to Theorem 1.

Theorem 3 A tight and compact extended formulation for $X^{2 D L S-(U, U)-S C}$ is given by:

$$
\begin{array}{ll}
s_{0}^{0}=\sum_{i \in I} \phi^{i}, & \\
\phi^{i}+s_{0}^{i}=\sum_{l=1}^{n} d_{l}^{i} \zeta_{l}^{i} & i \in I, \\
s_{0}^{i}=\sum_{l=1}^{n} d_{l}^{i} \delta_{l}^{i} & i \in I, \\
\zeta_{l}^{i} \geq \delta_{l}^{i} & i \in I, l \in[1, n], \\
\zeta_{l}^{i}+y_{1}^{i}+z_{2, l}^{i} \geq 1 & i \in I, l \in[1, n], \\
\zeta_{l}^{i}+y_{1}^{0}+z_{2 t}^{0}+y_{t+1}^{i}+z_{t+2, l}^{i} \geq 1 & i \in I, t \in[1, l-1], l \in[1, n], \\
\zeta_{l}^{i}+y_{1}^{0}+z_{2 l}^{0} \geq 1 & i \in I, l \in[1, n], \\
\delta_{l}^{i}+y_{1}^{i}+z_{2 l}^{i} \geq 1 & i \in I, l \in[1, n], \\
z_{j}^{i} \geq y_{j}^{i}-y_{j-1}^{i} & i \in I_{0}, j \in[1, n], \\
z_{j}^{i} \leq y_{j}^{i} & i \in I_{0}, j \in[1, n], \\
\zeta, \delta \in \mathbb{R}_{+}^{m n}, y \in \mathbb{R}_{+}^{(m+1)(n+1)}, z \in \mathbb{R}_{+}^{(m+1) n} . &
\end{array}
$$

As above, one can also obtain a formulation with an order of magnitude less constraints, the convex hull in the original $(s, y, z)$ space and a $\Theta(m n)$ separation algorithm. 


\subsection{Constant capacities for final products $2 D L S-(U, C C)$}

Here we suppose that $Q_{j}^{0}=M$ and $Q_{j}^{i}=Q^{i}$ for all $j \in[1, n]$ and all $i \in I$. As one again has $y^{0}=e_{\alpha}$ for some $\alpha \in[1, n+1]$ in all extreme points, we define the sets

$$
X^{\alpha}=X^{2 D L S-(U, C C)} \cap\left\{y^{0}: y_{1, \alpha-1}^{0}=0, y_{\alpha}^{0} \geq 1\right\},
$$

so the problem decomposes into $n+1$ subproblems

$$
X^{2 D L S-(U, C C)}=\bigcup_{\alpha=1}^{n+1} X^{\alpha}
$$

Our goal now is to describe $\operatorname{conv}\left(X^{\alpha}\right)$. Combined with the classical result of Balas [4] this will lead to a description of $\operatorname{conv}\left(X^{2 D L S-(U, C C)}\right)$.

Here we will encounter several sets of the form $X^{M I X}=\left\{(v, w) \in \mathbb{R}_{+}^{1} \times \mathbb{Z}_{+}^{n}\right.$ : $\left.v+w_{t} \geq b_{t} t \in[1, n]\right\}$, known as a mixing set [12]. The standard approach to obtain an extended formulation of such sets (see [22] Section 8.3.4 and [9]) uses the observation that in an extreme point, the fractional values $v \bmod 1$ must take either the value 0 , or one of the $n$ fractional values $b_{t} \bmod 1$. This forms the basis of an extended formulation for the convex hull in the form of a network dual matrix with integer right hand-sides whose size is linear in $n$.

Suppose now that $y^{0}$ is fixed. The set $X^{\alpha}$ decomposes by item giving $X^{\alpha}=$ $\bigcap_{i \in I} X^{\alpha, i}$, where $X^{\alpha, i}$ is the set:

$$
\begin{aligned}
& y_{1, \alpha-1}^{0}=0, \\
& y_{\alpha}^{0} \geq 1, \\
& \phi^{i}+s_{0}^{i} \geq d_{1, \alpha-1}^{i}, \\
& \phi^{i}+s_{0}^{i}+Q^{i} y_{\alpha l}^{i} \geq d_{1 l}^{i} \quad l \in[\alpha, n], \\
& s_{0}^{i}+Q^{i} y_{1 l}^{i} \geq d_{1 l}^{i} \quad l \in[1, n], \\
& \phi^{i}, s_{0}^{i} \geq 0, y^{i} \in\{0,1\}^{n} .
\end{aligned}
$$

To describe $\operatorname{conv}\left(X^{\alpha, i}\right)$, we suppose without loss of generality that $Q^{i}=1$, and we observe that $X^{\alpha, i}$ is essentially the intersection of two mixing sets, the first having the continuous variable $v=\phi^{i}+s_{0}^{i}$ and integer variables $w_{l}=y_{\alpha l}^{i}$ satisfying (58), (59) and bounds $y^{i} \in\{0,1\}^{n}$ and the second $v=s_{0}^{i}$, and $w_{l}=y_{1 l}^{i}$ satisfying (60) and $y^{i} \in\{0,1\}^{n}$. Here we observe that the fractional values $\phi^{i}+s_{0}^{i}$ and $s_{0}^{i}$ mod 1 must take either the value 0 , or one of the $n$ fractional values $d_{1 l}^{i} \bmod 1$. Let $f_{1}>f_{2}>\cdots>f_{\hat{n}}$ represent these distinct fractional parts in decreasing order, set $f_{0}=1$ and $f_{\hat{n}+1}=0$, and let $\pi(l)$ be the index in $[1, \hat{n}]$ with $f_{\pi(l)} \equiv d_{1 l}^{i} \bmod 1$ for $l \in[1, n]$.

Dropping the superscript $i$, introducing $\bar{y}_{t}=y_{1 t}$ and noting that $\bar{y}_{t}-\bar{y}_{\alpha-1}=y_{\alpha t}$, the network dual extended formulation for the two mixing sets gives: 


$$
\begin{array}{ll}
\phi+s_{0}=\sum_{l=0}^{\hat{n}}\left(f_{l}-f_{l+1}\right) \mu_{l}^{0}, & \\
\mu_{\pi(\alpha-1)}^{0} \geq\left\lfloor d_{1, \alpha-1}\right\rfloor+1, & \\
\mu_{\pi(l)}^{0}+\bar{y}_{l}-\bar{y}_{\alpha-1} \geq\left\lfloor d_{1 l}\right\rfloor+1 & \\
\mu_{\hat{n}}^{0}-\mu_{0}^{0}=1, & l \in[\alpha, n], \hat{n}], \\
\mu_{l}^{0}-\mu_{l-1}^{0} \geq 0 & \\
\mu_{0}^{0} \geq 0, & \\
s_{0}=\sum_{l=0}\left(f_{l}-f_{l+1}\right) \mu_{l}, & \\
\mu_{\pi(l)}+\bar{y}_{l} \geq\left\lfloor d_{1 l}\right\rfloor+1 & l \in[1, n], \\
\mu_{\hat{n}}-\mu_{0}=1, & l \in[1, \hat{n}], \\
\mu_{l}-\mu_{l-1} \geq 0 & l \in[1, n], \\
\mu_{0} \geq 0, & l \in[0, \hat{n}], \\
0 \leq \bar{y}_{l}-\bar{y}_{l-1} \leq 1 & \\
\mu_{l}^{0}-\mu_{l} \geq 0 & \\
\bar{y}_{0}=0 . &
\end{array}
$$

Here (62)-(67) is an extended formulation for the first mixing set, (68)-(72) is an extended formulation for the second, and (74) is a constraint linking the continuous variables $\phi^{i}+s_{0}^{i}$ and $s_{0}^{i}$ of the two mixing sets.

Consider now the matrix corresponding to the constraints (63)-(75), and call the associated polyhedron $P^{\alpha, i}$. The constraint matrix is not TU because of (74), but we can show integrality as follows.

We first show that the constraint matrix of (63)-(73) is TU, using again the characterization in [11]. Given a subset $J$ of variables, we put all variables $\bar{y}_{l}$ for $l \in[\alpha, n]$ in $J_{1}$ and all variables $\mu_{l}$ in $J_{2}$. If $\bar{y}_{\alpha-1}$ is in the set $J$, then we put $\bar{y}_{\alpha-1}$ and all variables $\mu_{l}^{0}$ in $J_{1}$. If $\bar{y}_{\alpha-1}$ is not in the set $J$, then we put all variables $\mu_{l}^{0}$ in $J_{2}$. It is easily checked that this partition satisfies the desired property.

Now, in extreme points of $P^{\alpha, i}$, for each $l$, either (74) is tight and $\mu_{l}^{0}=\mu_{l}$ implying that (67) is dominated by (64), so that (67) and therefore (74) can be dropped, or (74) itself can be dropped. In either cases, we have just shown that the resulting system of inequalities is TU. Therefore each extreme point of $P^{\alpha, i}$ is contained in a face that is itself an integral polyhedron and thus $P^{\alpha, i}$ is an integral polyhedron.

We have obtained a description of $\operatorname{conv}\left(X^{\alpha}\right)$ :

$$
\begin{aligned}
y_{1, \alpha-1}^{0} & =0, y_{\alpha}^{0} \geq 1, \\
\left(\phi^{i}, s^{i}, y^{i}\right) & \in P^{\alpha, i} i \in I,
\end{aligned}
$$

which can then be written compactly as the polyhedron

$$
F^{\alpha}(s, y, \phi) \geq g^{\alpha} .
$$


Theorem 4 An extended formulation for $\operatorname{conv}\left(X^{2 D L S-(U, C C)}\right)$ is given by:

$$
\begin{array}{ll}
s_{0}^{0}=\sum_{i \in I} \phi^{i}, & \\
y^{i}=\sum_{\alpha=1}^{n+1} y^{i, \alpha} & i \in I_{0}, \\
\phi^{i}=\sum_{\alpha=1}^{n+1} \phi^{i, \alpha} & i \in I, \\
s_{0}^{i}=\sum_{\alpha=1}^{n+1} s^{i, \alpha} & \\
F^{\alpha}\left(s^{., \alpha}, y^{, \alpha}, \phi^{., \alpha}\right) \geq g^{\alpha} \omega_{\alpha} & \\
n+1 & \\
\sum_{\alpha=1}^{n+1} \omega_{\alpha}=1, & \\
\omega \in \mathbb{R}_{+}^{n+1} . &
\end{array}
$$

\subsection{Production capacities at both levels}

Here we assume that the production capacity is identical at both levels and for all items, i.e., $Q^{i}=Q$ for all $i \in I_{0}$. Alternatively, one can take $Q=\max _{i \in I_{0}} Q^{i}$ to build such a relaxation.

Let $X^{i}=\left\{\left(\phi^{i}, s_{0}^{i}, y^{0}, y^{i}\right) \in \mathbb{R}_{+}^{2} \times\{0,1\}^{2 n}: \phi^{i}+s_{0}^{i}+Q y_{1 t}^{0}+Q y_{t+1, l}^{i} \geq d_{1 l}^{i}\right.$ for $l \in$ $[1, n], t \in[0, l]\}$. Note that if we set $z_{l}=\min _{t \in[0, l]}\left(y_{1 t}^{0}+y_{t+1, l}^{i}\right) \in \mathbb{Z}_{+}^{1}, s=\phi^{i}+s_{0}^{i}$, and $Y_{l}^{0}=y_{1 l}^{0}$, we obtain a mixing set plus additional constraints:

$$
\begin{array}{cc}
s+Q z_{l} \geq d_{1 l}^{i} & l \in[1, n], \\
z_{l} \leq Y_{l}^{0} & l \in[1, n], \\
z_{l} \leq z_{l-1}+y_{l}^{i} & l \in[1, n], \\
0 \leq Y_{l}^{0}-Y_{l-1}^{0} \leq 1 & l \in[1, n] \\
s \in \mathbb{R}_{+}, z \in \mathbb{Z}_{+}^{n}, Y^{0} \in \mathbb{Z}_{+}^{n}, y^{i} \in\{0,1\}^{n}, z_{0}=0 . &
\end{array}
$$

From [9] and as seen above in the formulation (62)-(75), an extended formulation of the mixing set $s+Q z_{l} \geq d_{1 l} l \in[1, n], s \in \mathbb{R}_{+}, z \in \mathbb{Z}_{+}^{n}$ is of the form $s=F \mu, A(z, \mu) \geq b$ where $A$ is a network dual matrix and $b$ is integer.

Proposition 5 The following is a tight and compact extended formulation for $X^{i}$.

$$
\begin{gathered}
s=F \mu, \\
A(z, \mu) \geq b,
\end{gathered}
$$




$$
\begin{array}{cl}
z_{l}-Y_{l}^{0} \leq 0 & l \in[1, n], \\
z_{l}-z_{l-1}-y_{l}^{i} \leq 0 & l \in[1, n], \\
0 \leq Y_{l}^{0}-Y_{l-1}^{0} \leq 1 & l \in[1, n], \\
s \in \mathbb{R}, z \in \mathbb{R}_{+}^{n}, Y^{0} \in \mathbb{R}_{+}^{n}, y^{i} \in[0,1]^{n} . &
\end{array}
$$

Proof Consider the matrix associated to constraints (84)-(88). Apart from the columns corresponding to the variables $y_{l}^{i}$ each of which appears only once, the remaining matrix is a network dual matrix, and hence TU. It follows that the complete matrix is TU. As the right hand sides and bounds are integer, the extended formulation is integral.

\section{Computational study}

\subsection{Computational results for the two-level lot-sizing problem with start-up costs}

In this section we report the results of our computational experiments for the two-level lot-sizing problem $(2 L S)$ with start-up costs. We performed tests with the original formulation (NF) (2)-(7) and (41), (42), the multicommodity formulation (MCF), see [24], and our extended formulation (EF) given in Theorem 3 and modified as in Observation 3. We also strengthened the natural formulation (NF-WW) and the multicommodity formulation (MCF-WW) with $(l, S)$ start-up inequalities [27] based on an echelon-stock reformulation, i.e., we used the inequalities

$$
\begin{aligned}
& s_{k-1}^{i} \geq \sum_{t=k}^{l} d_{t}^{i}\left(1-y_{k}^{i}-z_{k+1, t}^{i}\right) \quad i \in I, k \in[1, n], l \in[k, n], \\
& \sum_{i \in I_{0}} s_{k-1}^{i} \geq \sum_{i \in I} \sum_{t=k}^{l} d_{t}^{i}\left(1-y_{k}^{0}-z_{k+1, t}^{0}\right) \quad k \in[1, n], l \in[k, n],
\end{aligned}
$$

and their disaggregated versions

$$
\begin{aligned}
& \hat{s}_{k-1, l}^{i} \geq d_{l}^{i}\left(1-y_{k}^{i}-z_{k+1, l}^{i}\right) \quad i \in I, k \in[1, n], l \in[k, n], \\
& \hat{s}_{k-1, l}^{0 i}+\hat{s}_{k-1, l}^{i} \geq d_{l}^{i}\left(1-y_{k}^{0}-z_{k+1, l}^{0}\right) \quad i \in I, k \in[1, n], l \in[k, n],
\end{aligned}
$$

for NF and MCF respectively, where $\hat{s}_{k-1, l}^{0 i}$ and $\hat{s}_{k-1, l}^{i}$ give the amount of items 0 and $i$ that are in the inventory at the end of period $k-1$ and that are used to satisfy the demand of item $i$ in period $l$.

We first solve problems with 40 final products and 36 periods. As we are not aware of benchmark instances, we generate the data randomly as follows. The setup, startup, and inventory holding costs are constant over time, so we drop the index $t$. The inventory holding costs for the final products are generated randomly as integers in the interval $[1,5]$ and the cost for item 0 is taken as the minimum of these costs. The 
Table 1 Results for the two-level lot-sizing problem $(2 L S)$ with start-up costs

\begin{tabular}{|c|c|c|c|c|c|c|}
\hline n.m. $\rho$ & Formulation & Solved & LP-gap & f-gap & Nodes & Time \\
\hline \multirow[t]{5}{*}{36.40 .1} & $\mathrm{NF}$ & 0 & 72.5 & 21.7 & 32424.3 & 600 \\
\hline & NF-WW & 0 & 3.3 & 1.8 & 377.6 & 600 \\
\hline & $\mathrm{MCF}$ & 0 & 23.0 & 37.8 & 275.0 & 600 \\
\hline & MCF-WW & 2 & 0.2 & 16.8 & 38.7 & 536.9 \\
\hline & $\mathrm{EF}$ & 7 & 0.1 & 0.04 & 45.9 & 294.9 \\
\hline \multirow[t]{5}{*}{36.40 .5} & $\mathrm{NF}$ & 0 & 72.3 & 23.8 & 34802.7 & 600 \\
\hline & NF-WW & 0 & 4.8 & 4.2 & 101.4 & 600 \\
\hline & $\mathrm{MCF}$ & 0 & 23.4 & 47.0 & 168.4 & 600 \\
\hline & MCF-WW & 9 & 0.1 & 3.8 & 2.1 & 172.7 \\
\hline & $\mathrm{EF}$ & 9 & 0.1 & 0.03 & 9.4 & 159.8 \\
\hline \multirow[t]{5}{*}{36.40 .10} & $\mathrm{NF}$ & 0 & 71.7 & 22.5 & 30190.2 & 600 \\
\hline & NF-WW & 0 & 5.0 & 4.8 & 151.5 & 600 \\
\hline & $\mathrm{MCF}$ & 0 & 23.4 & 49.6 & 93.2 & 600 \\
\hline & MCF-WW & 9 & 0.03 & 0.01 & 3.0 & 182.2 \\
\hline & $\mathrm{EF}$ & 9 & 0.03 & 0.02 & 9.5 & 174.8 \\
\hline
\end{tabular}

demands are generated as integers in the interval [1,50]. For each item $i \in I_{0}$, we generated an integer $\hat{q}^{i}$ in the interval $[11,20]$. We use a parameter $\rho \in\{1,5,10\}$ to obtain instances with a different ratio of setup and start-up costs between the two levels. We set the start-up costs $\bar{q}_{i}$ and the setup costs $q_{i}$ as $\bar{q}^{i}=q^{i}=100 \hat{q}^{i}$ for $i \in I$ and $\bar{q}^{0}=q^{0}=100 \rho \hat{q}^{0}$.

All experiments are carried out using Xpress-IVE version 1.22.04 on a notebook with $2.20 \mathrm{GHz}$ Intel core 7 -2720QM processor and 8 GB RAM. The time limit is 600 s. For each $\rho$ value, we solve ten instances and report the average results. We report the number of instances solved to optimality, the gap of the LP relaxation (LPgap, computed using the best upper bound), the gap at termination (f-gap, computed using the upper and lower bounds at termination), the number of nodes explored, and the solution time in seconds. The results are presented in Table 1.

We observe that NF and MCF have huge duality gaps and adding the $(l, S)$ startup inequalities results in a considerable improvement. MCF-WW and EF have very similar duality gaps, but, more instances are solved to optimality with EF and the final gaps for those that are not solved are smaller. The results of this first experiment suggest that we may be able to compute good bounds for larger instances using NFWW, MCF-WW and EF. This is what we test in our second experiment.

In Table 2, we present results for instances with 40 final products and up to 60 periods and also for instances with 36 periods and up to 200 final products. Here we set $\rho=10$. We report the individual results rather than the averages. For each instance and formulation, we report the best lower and upper bounds and the gap on termination (BLB, BIP, and f-gap, respectively) when the time limit is set to 600 and 1,800 s respectively. If an instance is solved to optimality, we report the solution time in parentheses in the column f-gap. We present the gap between the best bounds in 
Table 2 Results for the two-level lot-sizing problem $(2 L S)$ with start-up costs-larger instances

\begin{tabular}{|c|c|c|c|c|c|c|c|c|c|}
\hline \multirow[t]{2}{*}{ n.m..$\rho$} & \multirow[t]{2}{*}{ Formulation } & \multicolumn{4}{|l|}{$600 \mathrm{~s}$} & \multicolumn{4}{|l|}{$1800 \mathrm{~s}$} \\
\hline & & BLB & BIP & f-gap & b-gap & BLB & BIP & f-gap & b-gap \\
\hline \multirow[t]{3}{*}{48.40 .10} & NF-WW & 1349460 & 1451110 & 7 & & 1350930 & 1416440 & 4.6 & \\
\hline & MCF-WW & 1409330 & 2454730 & 42.6 & 0.6 & 1409450 & 1414050 & 0.3 & 0.1 \\
\hline & $\mathrm{EF}$ & 1409210 & 1417880 & 0.6 & & 1411290 & 1412790 & 0.1 & \\
\hline \multirow[t]{3}{*}{48.40 .10} & NF-WW & 1274450 & 1357290 & 6.1 & & 1276300 & 1357290 & 6 & \\
\hline & MCF-WW & 1324120 & 2445830 & 45.9 & 2.4 & 1324480 & 2445830 & 45.8 & 0.9 \\
\hline & $\mathrm{EF}$ & 1324070 & 1472410 & 10.1 & & 1324070 & 1336700 & 0.9 & \\
\hline \multirow[t]{3}{*}{48.40 .10} & NF-WW & 1325560 & 1428630 & 7.2 & & 1326720 & 1392960 & 4.8 & \\
\hline & MCF-WW & 1384090 & 2453730 & 43.6 & 0.8 & 1384150 & 1388280 & 0.3 & 0.2 \\
\hline & $\mathrm{EF}$ & 1383990 & 1395130 & 0.8 & & 1386010 & 1388280 & 0.2 & \\
\hline \multirow[t]{3}{*}{48.40 .10} & NF-WW & 1332280 & 1412900 & 5.7 & & 1333110 & 1412900 & 5.6 & \\
\hline & MCF-WW & 1378670 & 2165870 & 36.3 & 0.0 & 1378760 & 1385540 & 0.5 & 0.0 \\
\hline & $\mathrm{EF}$ & 1379190 & 1379190 & (528) & & 1379190 & 1379190 & (528) & \\
\hline \multirow[t]{3}{*}{48.40 .10} & NF-WW & 1287260 & 1382810 & 6.9 & & 1288410 & 1382810 & 6.8 & \\
\hline & MCF-WW & 1341360 & 2321460 & 42.2 & 0.8 & 1341810 & 1345560 & 0.3 & 0.2 \\
\hline & $\mathrm{EF}$ & 1341310 & 1352080 & 0.8 & & 1342470 & 1346420 & 0.3 & \\
\hline \multirow[t]{3}{*}{60.40 .10} & NF-WW & 1669780 & 1827900 & 8.6 & & 1672080 & 1811510 & 7.7 & \\
\hline & MCF-WW & 1752500 & 3198990 & 45.2 & 4.1 & 1752530 & 3198990 & 45.2 & 3.3 \\
\hline & $\mathrm{EF}$ & 1752470 & 1862580 & 5.9 & & 1752470 & 1862580 & 5.9 & \\
\hline \multirow[t]{3}{*}{60.40 .10} & NF-WW & 1576590 & 1825690 & 13.6 & & 1579090 & 1705100 & 7.4 & \\
\hline & MCF-WW & 1646230 & 3010200 & 45.3 & 6.9 & 1646230 & 3010200 & 45.3 & 1.3 \\
\hline & $\mathrm{EF}$ & 1646220 & 1767980 & 6.9 & & 1646220 & 1667360 & 1.3 & \\
\hline \multirow[t]{3}{*}{60.40 .10} & NF-WW & 1633040 & 1782480 & 8.4 & & 1634240 & 1774300 & 7.9 & \\
\hline & MCF-WW & 1707610 & 3491780 & 51.1 & 4.2 & 1707620 & 3410900 & 49.9 & 3.8 \\
\hline & $\mathrm{EF}$ & 1707550 & 1891620 & 9.7 & & 1707550 & 1801350 & 5.2 & \\
\hline \multirow[t]{3}{*}{60.40 .10} & NF-WW & 1647810 & 1947920 & 15.4 & & 1649670 & 1763390 & 6.4 & \\
\hline & MCF-WW & 1712240 & 3144520 & 45.5 & 9.0 & 1712240 & 3144520 & 45.5 & 1.2 \\
\hline & $\mathrm{EF}$ & 1712200 & 1881210 & 9 & & 1712200 & 1733870 & 1.2 & \\
\hline \multirow[t]{3}{*}{60.40 .10} & NF-WW & 1602000 & 2014870 & 20.5 & & 1602770 & 1737130 & 7.7 & \\
\hline & MCF-WW & 1676800 & 3129720 & 46.4 & 6.0 & 1676820 & 3123790 & 46.3 & 3.5 \\
\hline & $\mathrm{EF}$ & 1676770 & 1783020 & 6 & & 1676770 & 1783020 & 6 & \\
\hline \multirow[t]{3}{*}{36.100 .10} & NF-WW & 2312990 & 2467330 & 6.3 & & 2322530 & 2467330 & 5.9 & \\
\hline & MCF-WW & 2406290 & 4174610 & 42.4 & 0.7 & 2406430 & 4174610 & 42.4 & 0.6 \\
\hline & $\mathrm{EF}$ & 2406280 & 2423230 & 0.7 & & 2406280 & 2421670 & 0.6 & \\
\hline \multirow[t]{3}{*}{36.100 .10} & NF-WW & 2223670 & 2360120 & 5.8 & & 2224780 & 2336010 & 4.8 & \\
\hline & MCF-WW & 2296800 & 3670860 & 37.4 & 0.6 & 2296800 & 3670860 & 37.4 & 0.2 \\
\hline & $\mathrm{EF}$ & 2298030 & 2311280 & 0.6 & & 2298420 & 2304050 & 0.2 & \\
\hline \multirow[t]{3}{*}{36.100 .10} & NF-WW & 2388690 & 2526500 & 5.5 & & 2390630 & 2526500 & 5.4 & \\
\hline & MCF-WW & 2473790 & 4185090 & 40.9 & 0.7 & 2473810 & 4185090 & 40.9 & 0.6 \\
\hline & $\mathrm{EF}$ & 2473980 & 2492370 & 0.7 & & 2474410 & 2490400 & 0.6 & \\
\hline
\end{tabular}


Table 2 continued

\begin{tabular}{|c|c|c|c|c|c|c|c|c|c|}
\hline \multirow[t]{2}{*}{ n.m. } & \multirow[t]{2}{*}{ Formulation } & \multicolumn{4}{|l|}{$600 \mathrm{~s}$} & \multicolumn{4}{|l|}{$1800 \mathrm{~s}$} \\
\hline & & BLB & BIP & f-gap & b-gap & BLB & BIP & f-gap & b-gap \\
\hline \multirow[t]{3}{*}{36.100 .10} & NF-WW & 2308170 & 2450650 & 5.8 & & 2312670 & 2442020 & 5.3 & \\
\hline & MCF-WW & 2387240 & 3822880 & 37.6 & 1.1 & 2387240 & 3822880 & 37.6 & 1.0 \\
\hline & $\mathrm{EF}$ & 2388630 & 2415370 & 1.1 & & 2388630 & 2412430 & 1 & \\
\hline \multirow[t]{3}{*}{36.100 .10} & NF-WW & 2358160 & 2491230 & 5.3 & & 2360680 & 2491230 & 5.2 & \\
\hline & MCF-WW & 2444620 & 2444620 & $(98)$ & 0.0 & 2444620 & 2444620 & (98) & 0.0 \\
\hline & $\mathrm{EF}$ & 2444620 & 2444620 & (130) & & 2444620 & 2444620 & (130) & \\
\hline \multirow[t]{3}{*}{36.200 .10} & NF-WW & 4478810 & 4970220 & 9.9 & & 4481750 & 4751750 & 5.7 & \\
\hline & MCF-WW & 4640070 & 7255190 & 36 & 5.9 & 4640080 & 7255190 & 36 & 2.3 \\
\hline & $\mathrm{EF}$ & 4643990 & 4936220 & 5.9 & & 4643990 & 4936220 & 5.9 & \\
\hline \multirow[t]{3}{*}{36.200 .10} & NF-WW & 4355180 & 4603180 & 5.4 & & 4358010 & 4565450 & 4.5 & \\
\hline & MCF-WW & 4474600 & 6335240 & 29.4 & 2.2 & 4474610 & 6335240 & 29.4 & 0.4 \\
\hline & $\mathrm{EF}$ & 4483970 & 4586380 & 2.2 & & 4483970 & 4501120 & 0.4 & \\
\hline \multirow[t]{3}{*}{36.200 .10} & NF-WW & 4461340 & 4886960 & 8.7 & & 4463550 & 4701160 & 5.1 & \\
\hline & MCF-WW & 4610080 & 7273270 & 36.6 & 4.7 & 4610090 & 7273270 & 36.6 & 0.8 \\
\hline & $\mathrm{EF}$ & 4614710 & 4841300 & 4.7 & & 4614710 & 4650590 & 0.8 & \\
\hline \multirow[t]{3}{*}{36.200 .10} & NF-WW & 4458110 & 4722690 & 5.6 & & 4461170 & 4665210 & 4.4 & \\
\hline & MCF-WW & 4579670 & 6682470 & 31.5 & 2.0 & 4579690 & 6682470 & 31.5 & 1.7 \\
\hline & $\mathrm{EF}$ & 4587820 & 4679750 & 2 & & 4587820 & 4679750 & 2 & \\
\hline \multirow[t]{3}{*}{36.200 .10} & NF-WW & 4517440 & 4981930 & 9.3 & & 4519460 & 4774180 & 5.3 & \\
\hline & MCF-WW & 4678910 & 7172630 & 34.8 & 3.8 & 4678930 & 7172630 & 34.8 & 0.9 \\
\hline & $\mathrm{EF}$ & 4683750 & 4868340 & 3.8 & & 4683750 & 4726940 & 0.9 & \\
\hline
\end{tabular}

column "b-gap". We observe that the solver usually finds good solutions with NFWW, however the lower bounds are significantly worse than those of the other two formulations. With MCF-WW, upper bounds are of poor quality and letting the solver run for half an hour only leads to an improvement for the instances with 48 periods and 40 products. Using EF, one may obtain good solutions with a less than $1 \%$ gap in 10 min when $n=48$, however the results are not good for $n=60$. If the number of periods is not large, EF remains the most efficient formulation for our instances with larger values of $m$.

4.2 Computational results for the two-level lot-sizing problem with constant capacities for final products

Now we present computational results for the capacitated lot-sizing problem where $Q^{0}=M$ and $Q^{i}=Q$ for all $i \in I$. Here, we compare again the natural formulation $(\mathrm{NF})$, the multicommodity formulation $(\mathrm{MCF})$, and our extended formulation (EF) (76)-(82). We also test NF and MCF with an approximation of the constant capacity Wagner-Whitin extended formulation $[21,28]$. We refer to the resulting formulations as NF-WW and MCF-WW. 
Table 3 Results for the discrete two-level lot-sizing problem (2DLS) with constant capacities for final products

\begin{tabular}{llrlrrl}
\hline n.m. $\rho$ & Formulation & Solved & LP-gap & f-gap & Nodes & Time \\
\hline 60.40 .1 & NF & 0 & 3.0 & 1.7 & 37842.2 & 180 \\
& NF-WW & 10 & 0.5 & 0.0 & 4.0 & 70.7 \\
& MCF & 0 & 1.5 & 1.5 & 427.7 & 180 \\
& MCF-WW & 3 & 0.1 & 5.8 & 0.3 & 180 \\
& EF & 10 & 0.0 & 0.0 & 1.0 & 96.9 \\
60.40 .5 & NF & 0 & 4.3 & 1.6 & 37675.0 & 180 \\
& NF-WW & 10 & 1.0 & 0.0 & 3.4 & 88 \\
& MCF & 0 & 1.4 & 1.5 & 336.3 & 180 \\
& MCF-WW & 1 & 0.4 & 14.2 & 0.1 & 180 \\
& EF & 10 & 0.0 & 0.0 & 1.0 & 94.6 \\
& NF & 0 & 4.9 & 1.6 & 37203.8 & 180 \\
& NF-WW & 10 & 1.0 & 0.0 & 5.6 & 102.5 \\
& MCF & 0 & 1.4 & 1.6 & 255.7 & 180 \\
& MCF-WW & 1 & 0.4 & 18.7 & 0.1 & 180 \\
& EF & 10 & 0.0 & 0.0 & 1.0 & 95.3 \\
\hline
\end{tabular}

In Table 3, we report the results for the discrete lot-sizing problem ( $2 D L S$, only the initial stock variables and setup variables have nonzero costs). Here we consider instances with 40 final products and 60 periods and take the costs for the initial stocks to be equal to 1 . The setup cost at level 0 in period $t$ is obtained by multiplying $\rho$ by an integer generated randomly in the interval $[50,50+20(n-t)]$ and for the other items, $q_{t}^{i}$ is randomly generated in the interval $[51,70]$. The demands are generated as integers in the interval $[1,50]$ and the capacity is taken to be 100 . The time limit is $180 \mathrm{~s}$. For each $\rho$ value, we report the averages for ten instances.

All instances are solved to optimality with formulations NF-WW and EF within the time limit. In most cases, NF-WW proves optimality sooner than EF.

The results for the two-level lot-sizing problem $(2 L S)$ are given in Table 4 . Here we take $n=18$ and $m=20$. The data is generated in the same way as for the instances with start-ups except that we set $q^{i}=200 \hat{q}^{i}$ for $i \in I$ and $q^{0}=200 \rho \hat{q}^{0}$. We take the capacity to be equal to 100 . In this experiment, the time limit is set to $600 \mathrm{~s}$. We report the average results for ten instances for each $\rho$ value. Here, it is clear that NF and MCF have large duality gaps and cannot obtain optimal solutions within the time limit. However, when strengthened, these formulations outperform EF in terms of computation time.

Due to its large size, EF takes longer to solve for larger instances. In our final experiment, we use NF-WW and MCF-WW to see the quality of bounds that one can obtain as $n$ and $m$ increase. The results are given in Table 5. Here the results are given for individual instances.

Except for the instances solved to optimality, the best lower bounds are obtained using MCF-WW and the best upper bounds using NF-WW. We see that the lower 
Table 4 Results for the two-level lot-sizing problem $(2 L S)$ with constant capacities for final products

\begin{tabular}{llrrrrr}
\hline n.m. & Formulation & Solved & LP-gap & f-gap & Nodes & Time \\
\hline 18.20 .1 & NF & 0 & 17.8 & 2.2 & 79294.6 & 600 \\
& NF-WW & 10 & 3.6 & 0.0 & 381.6 & 13.5 \\
& MCF & 0 & 4.7 & 5.5 & 44335.3 & 600 \\
& MCF-WW & 10 & 0.3 & 0.0 & 95.0 & 23.8 \\
& EF & 10 & 0.0 & 0.0 & 1.4 & 108.8 \\
& NF & 0 & 18.5 & 3.0 & 78988.2 & 600 \\
& NF-WW & 10 & 5.5 & 0.0 & 71.4 & 8.1 \\
& MCF & 0 & 4.1 & 4.1 & 48509.6 & 600 \\
& MCF-WW & 10 & 0.3 & 0.0 & 175.2 & 30.8 \\
& EF & 10 & 0.0 & 0.0 & 1.0 & 94.4 \\
& NF & 0 & 18.1 & 2.9 & 79357.1 & 600 \\
& NF-WW & 10 & 6.5 & 0.0 & 37.6 & 7.7 \\
& MCF & 0 & 3.4 & 3.7 & 56635.1 & 600 \\
& MCF-WW & 10 & 0.2 & 0.0 & 53.8 & 12.3 \\
& EF & 10 & 0.0 & 0.0 & 1.0 & 54.1 \\
\hline
\end{tabular}

bounds obtained by MCF-WW in half an hour are very close to those obtained after $10 \mathrm{~min}$. However, for several instances, there was a significant improvement in the upper bounds obtained with NF-WW after half an hour. Overall, we obtain good solutions with small duality gaps even for problems with 24 periods and 200 final products in half an hour using NF-WW and compute good lower bounds in $10 \mathrm{~min}$ using MCF-WW.

\section{Conclusions}

In this paper, we have proposed exact and approximate extended formulations for two-level multi-item discrete lot-sizing problems and reported some computational results on using these reformulations to solve one-producer multiple item lot-sizing, or equivalently one-warehouse multiple-retailer problems. We have proposed an exact extended formulation for the uncapacitated problem and modified it to handle start-up costs. In our computational experiments, we have observed that the extended formulation for the problem with start-up costs outperforms the existing formulations. We note that this formulation can be extended easily to problems with more levels and to problems with demand at intermediate levels.

We have also proposed an exact extended formulation for the problem with constant capacities for final products and no capacity constraints at the upper level. Here the behavior of the formulations appears to be different. Even though the LP relaxation of the extended formulation has a duality gap smaller than those of the existing formulations, it is impractical due to its large size. One interesting extension of the current work may be to study the projection of this large formulation onto the space 
Table 5 Results for the two-level lot-sizing problem $(2 L S)$ with constant capacities for final productslarger instances

\begin{tabular}{|c|c|c|c|c|c|c|c|c|c|}
\hline \multirow[t]{2}{*}{ n.m. } & \multirow[t]{2}{*}{ Formulation } & \multicolumn{4}{|l|}{$600 \mathrm{~s}$} & \multicolumn{4}{|l|}{$1800 \mathrm{~s}$} \\
\hline & & BLB & BIP & f-gap & b-gap & BLB & BIP & f-gap & b-gap \\
\hline \multirow[t]{2}{*}{36.40 .10} & NF-WW & 1529920 & 1567150 & 2.4 & 0.9 & 1566780 & 1566780 & (1430.4) & 0.0 \\
\hline & MCF-WW & 1553790 & 1593010 & 2.5 & & 1553940 & 1593010 & 2.5 & \\
\hline \multirow[t]{2}{*}{36.40 .10} & NF-WW & 1462850 & 1524370 & 4 & 1.6 & 1475020 & 1516800 & 2.8 & 1.1 \\
\hline & MCF-WW & 1500190 & 1851220 & 19 & & 1500190 & 1851220 & 19 & \\
\hline \multirow[t]{2}{*}{36.40 .10} & NF-WW & 1520540 & 1572720 & 3.3 & 1.4 & 1550010 & 1568810 & 1.2 & 1.1 \\
\hline & MCF-WW & 1551420 & 1669520 & 7.1 & & 1551840 & 1669520 & 7 & \\
\hline \multirow[t]{2}{*}{36.40 .10} & NF-WW & 1551350 & 1597730 & 2.9 & 1.2 & 1572680 & 1597130 & 1.5 & 1.2 \\
\hline & MCF-WW & 1578070 & 1926930 & 18.1 & & 1578470 & 1926930 & 18.1 & \\
\hline \multirow[t]{2}{*}{36.40 .10} & NF-WW & 1508100 & 1613960 & 6.6 & 2.3 & 1542720 & 1598140 & 3.5 & 1.3 \\
\hline & MCF-WW & 1576950 & 1869340 & 15.6 & & 1576950 & 1617880 & 2.5 & \\
\hline \multirow[t]{2}{*}{48.40 .10} & NF-WW & 2019110 & 2151720 & 6.2 & 3.1 & 2042670 & 2111810 & 3.3 & 1.3 \\
\hline & MCF-WW & 2084130 & 2802840 & 25.6 & & 2084310 & 2802840 & 25.6 & \\
\hline \multirow[t]{2}{*}{48.40 .10} & NF-WW & 1947730 & 2082490 & 6.5 & 3.3 & 1968400 & 2066990 & 4.8 & 2.5 \\
\hline & MCF-WW & 2014520 & 2781850 & 27.6 & & 2014760 & 2781850 & 27.6 & \\
\hline \multirow[t]{2}{*}{48.40 .10} & NF-WW & 1992340 & 2131210 & 6.5 & 2.9 & 1993710 & 2131210 & 6.5 & 2.9 \\
\hline & MCF-WW & 2070010 & 2815940 & 26.5 & & 2070090 & 2815940 & 26.5 & \\
\hline \multirow[t]{2}{*}{48.40 .10} & NF-WW & 2042270 & 2145710 & 4.8 & 2.9 & 2049970 & 2136760 & 4.1 & 2.5 \\
\hline & MCF-WW & 2083590 & 2899160 & 28.1 & & 2083590 & 2899160 & 28.1 & \\
\hline \multirow[t]{2}{*}{48.40 .10} & NF-WW & 2028550 & 2202880 & 7.9 & 4.5 & 2043390 & 2150210 & 5 & 2.1 \\
\hline & MCF-WW & 2104500 & 2544240 & 17.3 & & 2104500 & 2544240 & 17.3 & \\
\hline \multirow[t]{2}{*}{18.200 .10} & NF-WW & 3441130 & 3520510 & 2.3 & 0.5 & 3517430 & 3517430 & (1378.5) & 0.0 \\
\hline & MCF-WW & 3502530 & 3839370 & 8.8 & & 3509910 & 3517420 & 0.2 & \\
\hline \multirow[t]{2}{*}{18.200 .10} & NF-WW & 3415680 & 3459820 & 1.3 & 0.1 & 3454160 & 3454160 & (1311) & 0.0 \\
\hline & MCF-WW & 3451890 & 3454160 & 0.1 & & 3454160 & 3454160 & (714.9) & \\
\hline \multirow[t]{2}{*}{18.200 .10} & NF-WW & 3479110 & 3538250 & 1.7 & 0.4 & 3537030 & 3537030 & (1251) & 0.0 \\
\hline & MCF-W & 3525260 & 3875020 & 9.0 & & 3526520 & 3537470 & 0.3 & \\
\hline \multirow[t]{2}{*}{18.200 .10} & NF-WW & 3461570 & 3511120 & 1.4 & 0.4 & 3509970 & 3509970 & (1588) & 0.0 \\
\hline & MCF-WW & 3498720 & 3525420 & 0.8 & & 3509970 & 3509970 & (1394) & \\
\hline \multirow[t]{2}{*}{18.200 .10} & NF-WW & 3516080 & 3586940 & 2 & 0.5 & 3581550 & 3581550 & (1505) & 0.0 \\
\hline & MCF-WW & 3568030 & 3600230 & 0.9 & & 3574310 & 3581550 & 0.2 & \\
\hline \multirow[t]{2}{*}{24.200 .10} & NF-WW & 4576260 & 4735010 & 3.4 & 1.0 & 4591450 & 4735010 & 3 & 1.0 \\
\hline & MCF-WW & 4687440 & 5491390 & 14.6 & & 4687440 & 4747670 & 1.3 & \\
\hline \multirow[t]{2}{*}{24.200 .10} & NF-WW & 4522320 & 4629380 & 2.3 & 0.8 & 4539390 & 4626280 & 1.9 & 0.7 \\
\hline & MCF-WW & 4593880 & 5333280 & 13.9 & & 4595210 & 5333280 & 13.8 & \\
\hline \multirow[t]{2}{*}{24.200 .10} & NF-WW & 4571460 & 4728550 & 3.3 & 1.1 & 4600860 & 4719830 & 2.5 & 0.9 \\
\hline & MCF-WW & 4678810 & 5484460 & 14.7 & & 4678810 & 4749740 & 1.5 & \\
\hline \multirow[t]{2}{*}{24.200 .10} & NF-WW & 4597600 & 4998060 & 8.0 & 6.4 & 4624760 & 4709410 & 1.8 & 0.7 \\
\hline & MCF-WW & 4677980 & 5395220 & 13.3 & & 4677980 & 4742970 & 1.4 & \\
\hline 24.200 .10 & NF-WW & 4634810 & 4791790 & 3.3 & 0.9 & 4671710 & 4791790 & 2.5 & 0.9 \\
\hline & MCF-WW & 4748100 & 5529970 & 14.1 & & 4749910 & 5529970 & 14.1 & \\
\hline
\end{tabular}


of the original variables and devise a branch-and-cut algorithm. For the more general problem in which capacity constraints are also introduced at the upper level, we have only provided an extended formulation for a relaxation. Testing the performance of this extended formulation in practice and finding an exact extended formulation for this version of the problem remain for further investigation.

Finally, we conjecture that the following is an exact extended formulation for the two-level discrete lot-sizing problem with a single final product and backlogging:

$$
\begin{array}{ll}
s^{0}+s^{1}=\sum_{l=1}^{n} d_{l} \zeta_{l}, & \\
s^{1}=\sum_{l=1}^{n} d_{l} \delta_{l}, & \\
r_{l}=\sum_{j=1}^{l} d_{j} \sigma_{j l} & l \in[1, n], \\
\zeta_{j}+\sigma_{j l} \geq 1-y_{1 t}^{0}-y_{t+1, l}^{1} & l \in[1, n], t \in[0, l], j \in[1, l], \\
\delta_{j}+\sigma_{j l} \geq 1-y_{1 l}^{1} & l \in[1, n], j \in[1, l], \\
\zeta, \delta, y^{0}, y^{1} \in \mathbb{R}_{+}^{n}, \sigma \in \mathbb{R}_{+}^{\frac{n(n-1)}{2}}, &
\end{array}
$$

where $r_{l}$ is the amount backlogged at the end of period $l$. The approach in Proposition 3 can then be used to extend this formulation to multiple final products.

\section{Appendix: Proof of Theorem 3}

Similar to Theorem 1, the proof is in three steps. In the first step, we show that the polyhedron $S C C$ defined by (49)-(55) is integral for $m=1$. Then we extend the result to $m>1$ and finally prove that adding constraints (48) does not destroy integrality.

Let $S S C_{I}$ denote the set of integral solutions in $S S C$ and consider the case $m=$ 1. As we did in the proof of Theorem 2, for a given non-zero objective function $\min \sum_{u=1}^{n} g_{u}^{0} \zeta_{u}+\sum_{u=1}^{n} g_{u}^{1} \delta_{u}+\sum_{i=0}^{1} \sum_{u=0}^{n} q_{u}^{i} y_{u}^{i}+\sum_{i=0}^{1} \sum_{u=1}^{n} \bar{q}_{u}^{i} z_{u}^{i}$ with bounded optimal value, we determine one inequality among (49)-(55) that is satisfied at equality by all optimal solutions.

We use the following observation. Let $\alpha_{1} \in[0, n+1], \alpha_{2} \in\left[\alpha_{1}, n+1\right], \beta_{2} \in$ $\left[\alpha_{1}, n+1\right], \beta_{1} \in\left[0, \beta_{2}\right]$ with $\beta_{1}=n+1$ if $\beta_{2}=n+1, \gamma_{2} \in\left[1, \alpha_{1}-1\right] \cup\left\{\beta_{2}\right\}$, $\gamma_{1} \in\left[0, \gamma_{2}\right]$ if $\gamma_{2} \leq \alpha_{1}-1$ and $\gamma_{1}=\beta_{1}$ if $\gamma_{2}=\beta_{2}$. The $y$ and $z$ vectors in the extreme points of $\operatorname{conv}\left(S S C_{I}\right)$ are of the following form: $y_{u}^{0}=1$ for $u \in\left[\alpha_{1}, \alpha_{2}\right]$, $z_{\alpha_{1}}^{0}=1, y_{u}^{1}=1$ for $u \in\left[\gamma_{1}, \gamma_{2}\right] \cup\left[\beta_{1}, \beta_{2}\right], z_{\gamma_{1}}^{1}=z_{\beta_{1}}^{1}=1$, the other entries of $y$ and $z$ vectors are zero. In the sequel, we use the values $\alpha_{1}, \alpha_{2}, \gamma_{1}, \gamma_{2}, \beta_{1}, \beta_{2}$ to represent the corresponding extreme points.
a. Let $\bar{q}_{0}^{0}=\bar{q}_{0}^{1}=0$. We need $g^{0}, g^{1} \geq 0, \bar{q}_{t}^{0}+\sum_{u=t}^{t+k} q_{u}^{0} \geq 0$ and $\bar{q}_{t}^{1}+\sum_{u=t}^{t+k} q_{u}^{1} \geq 0$ for all $t \in[0, n], k \in[0, n-t]$ for the problem to be bounded.
b. For $i=0,1$, if there exists $u \in[1, n]$ with $q_{u}^{i}<0$, then $y_{u}^{i}=z_{u}^{i}+y_{u-1}^{i}$. 
c. For $i=0,1$, if there exists $u \in[1, n]$ with $\bar{q}_{u}^{i}<0$, then $z_{u}^{i}=y_{u}^{i}$.

d. For $i=0,1$, if $q_{0}^{i}<\bar{q}_{1}^{i}$, then $z_{1}^{i}=0$. If $q_{0}^{i}>\bar{q}_{1}^{i}$, then $y_{0}^{i}=0$. So $q_{0}^{i}=\bar{q}_{1}^{i}$. In the remaining, we study the case where $g^{0}, g^{1}, q^{0}, q^{1}, \bar{q}^{0}, \bar{q}^{1} \geq 0$.

e. If $g^{0}=q^{0}=\bar{q}^{0}=0$, then the problem is single-level and the result is known to hold [21].

f. Suppose that $g^{0}=0$. If $q_{0}^{0}>0$, then $y_{0}^{0}=0$. If there exists $u \in[1, n]$ with $q_{u}^{0}+\bar{q}_{u}^{0}>0$, then $z_{u}^{0}=0$. In the remaining, we assume that there exists $l$ such that $g_{l}^{0}>0$. Let $l$ be the highest such index.

g. If $q_{u}^{0}+\bar{q}_{u}^{0}<q_{u+1}^{0}+\bar{q}_{u+1}^{0}$ for some $u \in[1, n-1]$, then $z_{u+1}^{0}=0$. Let $t \in[1, n]$ be the largest index with $q_{t}^{0}+\bar{q}_{t}^{0}>0$. If no such $t$ exists, then let $t=0$. If $t>l$, then $z_{t}^{0}=0$. So we assume that $t \leq l$.

h. If there exist $k \in[1, l], m_{1} \in[0, \bar{k}]$ and $m_{2} \in[k, n]$ such that $\bar{q}_{k}^{0}+q_{k}^{0}+\bar{q}_{m_{1}}^{1}+$ $\sum_{u=m_{1}}^{m_{2}} q_{u}^{1}<g_{l}^{0}$, then $\zeta_{l}=0$. Therefore, as $\bar{q}_{k}^{0}+q_{k}^{0}=0$ for $k>t$, we assume that $\bar{q}_{m_{1}}^{1}+\sum_{u=m_{1}}^{m_{2}} q_{u}^{1} \geq g_{l}^{0}>0$ for all $m_{2} \in[t+1, n]$ and $m_{1} \in\left[0, m_{2}\right]$.

i. If $q_{t+1}^{1}>0$, we show that with $t, l$ chosen in this way, the inequality (49) if $t=0$, (50) if $t \in[1, l-1]$ or (51) if $t=l$ is satisfied at equality by all optimal solutions. Note that the cost assumptions imply that all rays with non-zero contribution in this inequality have positive cost.

Let $\left(\zeta, \delta, y^{0}, y^{1}, z^{0}, z^{1}\right)$ be an extreme point optimal solution. Suppose that the inequality (49), (50), or (51) corresponding to the above choice of $t$ and $l$ is not tight.

(a) Case $\zeta_{l}=1$.

If $y_{t+1}^{1}+\sum_{u=t+2}^{l} z_{u}^{1} \geq 1$, then $t+1 \leq \beta_{2}$ and $\sum_{u=1}^{t} y_{u}^{0}=0$. In this case, setting $\alpha_{1}, \alpha_{2} \leftarrow t+1$ and $\zeta_{l}=0$ decreases the cost by $g_{l}^{0}>0$.

If $y_{t+1}^{1}+\sum_{u=t+2}^{l} z_{u}^{1}=0$, then $y_{1}^{0}+\sum_{u=2}^{t} z_{u}^{0}=1$ and $\beta_{1}>l$. Now, setting $\alpha_{1}, \alpha_{2} \leftarrow t+1$ yields a better solution since $q_{u}^{0}+\bar{q}_{u}^{0}>0$ for all $u \in[1, t]$ and $q_{0}^{0}=\bar{q}_{1}^{0}$.

(b) Case $\zeta_{l}=0$.

If $y_{1}^{0}+\sum_{u=2}^{t} z_{u}^{0}=0$, then $y_{t+1}^{1}+\sum_{u=t+2}^{l} z_{u}^{1} \geq 2$. Hence $t+1 \leq \gamma_{2}$ and $\beta_{1} \geq t+2$. Now setting $\alpha_{1}, \alpha_{2} \leftarrow \gamma_{2}, \beta_{1} \leftarrow \gamma_{1}$ and $\beta_{2} \leftarrow \gamma_{2}$ decreases the cost by $\bar{q}_{\beta_{1}}+\sum_{u=\beta_{1}}^{\beta_{2}} q_{u}^{1}$, which is positive since $\beta_{1} \in[t+2, l]$.

If $y_{1}^{0}+\sum_{u=2}^{t} z_{u}^{0}=1$, then $y_{t+1}^{1}+\sum_{u=t+2}^{l} z_{u}^{1} \geq 1$. If $\beta_{1} \geq t+1$, then setting $\alpha_{1}, \alpha_{2} \leftarrow t+1$ gives a better solution. If $\beta_{1} \leq t$, then $y_{t+1}^{1}=1$ and $\sum_{u=t+1}^{\beta_{2}} q_{u}^{1}>0$, so it is better to set $\beta_{2} \leftarrow t$.

j. If $g_{t}^{0}>0$ (and $q_{t+1}^{1}=0$, but this is not necessary here), then inequality $\zeta_{t} \geq 1-y_{1}^{0}-\sum_{u=2}^{t} z_{u}^{0}$ (i.e., of type (51)) is satisfied at equality. Indeed, if not, then $\zeta_{t}=1, y_{1}^{0}+\sum_{u=2}^{t} z_{u}^{0}=1$, and $\beta_{1} \geq t+1$. Then setting $\alpha_{1}, \alpha_{2} \leftarrow t+1$ gives a better solution.

k. If $q_{t+1}^{1}=0$ and $g_{t}^{0}=0$, then $z_{t}^{0}=0$, or equivalently $\alpha_{1} \neq t$, in any optimal solution. Indeed, if $\alpha_{1}=t$ and $\beta_{2}>t$, then setting $\alpha_{1}, \alpha_{2} \leftarrow t+1$ gives a better solution. If $\alpha_{1}=t$ and $\beta_{2}=t$, then setting $y_{t+1}^{1}=1$ at zero cost (and therefore $\left.\beta_{2}=t+1\right)$ and $\alpha_{1}, \alpha_{2} \leftarrow t+1$ gives a better solution.

Thus we can conclude that all optimal solutions lie on a face defined by one of the inequalities (49)-(55). This proves that $S S C$ is integral when $m=1$. 
To prove that the result is true for $m>1$, we need a variant of Proposition 3. First, we observe that in an extreme point of $\operatorname{conv}\left(S S C_{I}\right)$, we have $y_{0}^{0}+\sum_{j=1}^{n} z_{j}^{0} \leq 1$ and that given any $\left(\zeta, \delta, y^{0}, y^{1}, z^{0}, z^{1}\right) \in S S C$, the solution $\left(\zeta, \delta, \bar{y}^{0}, y^{1}, \bar{z}^{0}, z^{1}\right)$ is also in $S S C$ where $\bar{y}_{0}^{0}=\min \left\{y_{0}^{0}, 1\right\}, \bar{y}_{1}^{0}=\min \left\{z_{1}^{0}+y_{0}^{0}, 1\right\}, \bar{z}_{j}^{0}=\min \left\{\left(1-y_{0}^{0}-z_{1 j-1}^{0}\right)^{+}, z_{j}^{0}\right\}$ for $j=1, \ldots, n$, and $\bar{y}_{j}^{0}=\bar{z}_{j}^{0}$ for $j=2, \ldots, n$. Now, we can use similar arguments to those of Proposition 3 to obtain the result.

Finally we need to show that adding constraints (48) does not destroy integrality. As in the proof of Theorem 1, the key argument is that when such an inequality is tight, constraint (52) is dominated.

\section{References}

1. Afentakis, P., Gavish, B.: Optimal lot-sizing algorithms for complex product structures. Oper. Res. 34, 237-249 (1986)

2. Aggarwal, A., Park, J.: Improved algorithms for economic lot-size problems. Oper. Res. 41, 549-571 (1993)

3. Arkin, E., Joneja, D., Roundy, R.: Computational complexity of uncapacitated multi-echelon production planning problems. Oper. Res. Lett. 8, 61-66 (1989)

4. Balas, E.: On the convex hull of the union of certain polyhedra. Oper. Res. Lett. 7(6), 279-283 (1988)

5. Barany, I., Edmonds, J., Wolsey, L.A.: Packing and covering a tree by subtrees. Combinatorica 6, 245-257 (1986)

6. Barany, I., Van Roy, T.J., Wolsey, L.A.: Uncapacitated lot sizing: the convex hull of solutions. Math. Program. 22, 32-43 (1984)

7. Bitran, G.R., Yanasse, H.H.: Computational complexity of the capacitated lot size problem. Manag. Sci. 28, 1174-1186 (1982)

8. Clark, A.J., Scarf, H.: Optimal policies for multi-echelon inventory problems. Manag. Sci. 6, 475-490 (1960)

9. Conforti, M., Di Summa, M., Eisenbrand, F., Wolsey, L.A.: Network formulations of mixed-integer programs. Math. Oper. Res. 34, 194-209 (2009)

10. Federgrün, A., Tzur, M.: A simple forward algorithm to solve general dynamic lot-size models with $n$ periods in $O(n \log n)$ or $O(n)$ time. Manag. Sci. 37, 909-925 (1991)

11. Ghouila-Houri, A.: Caracterisation des matrices totalement unimodulaires. C.R. Acad. Sci. Paris 254, 1192-1194 (1962)

12. Günlük, O., Pochet, Y.: Mixing mixed integer inequalities. Math. Program. 90, 429-457 (2001)

13. Hwang, H.-C.: Economic lot-sizing for integrated production and transportation. Oper. Res. 58(2), 428-444 (2010)

14. Krarup, J., Bilde, O.: Plant location, set covering and economic lot sizes: an $O(m n)$ algorithm for structured problems. In: Collatz, L., et al., (eds.) Optimierung bei Graphentheoretischen und Ganzzahligen Probleme, pp. 155-180. Birkhauser Verlag, Basel (1977)

15. Küçükyavuz, S., Pochet, Y.: Uncapacitated lot-sizing with backlogging: the convex hull. Math. Program. 118, 151-175 (2009)

16. Lovasz, L.: Graph theory and integer programming. Ann. Discret. Math. 4, 141-158 (1079)

17. Melo, R., Wolsey, L.A.: Uncapacitated two-level lot-sizing. Oper. Res. Lett. 38, 241-245 (2010)

18. Pochet, Y., Wolsey, L.A.: Lot-size models with backlogging: strong formulations and cutting planes. Math. Program. 40, 317-335 (1988)

19. Pochet, Y., Wolsey, L.A.: Solving multi-item lot-sizing problems using strong cutting planes. Manag. Sci. 37(1), 53-67 (1991)

20. Pochet, Y., Wolsey, L.A.: Lot-sizing with constant batches: formulation and valid inequalities. Math. Oper. Res. 18, 767-785 (1993)

21. Pochet, Y., Wolsey, L.A.: Polyhedra for lot-sizing with Wagner-Whitin costs. Math. Program. 67, 297-324 (1994)

22. Pochet, Y., Wolsey, L.A.: Production Planning by Mixed Integer Programming. Springer, Berlin (2006)

23. Pochet, Y., Wolsey, L.A.: Single item lot-sizing with non-decreasing capacities. Math. Program. 121, 123-143 (2010) 
24. Rardin, R.L., Choe, U.: Tighter relaxations of fixed charge network flow problems. Technical Report report J-79-18, School of Industrial and Systems Engineering, Georgia Institute of Technology (1979)

25. van Hoesel, C.P.M., Romijn, H.E., Romero Morales, D., Wagelmans, A.: Integrated lot-sizing in serial supply chains with production capacities. Manag. Sci. 51, 1706-1719 (2005)

26. van Hoesel, C.P.M., Wagelmans, A., Moerman, B.: Using geometric techniques to improve dynamic programming algorithms for the economic lot-sizing problem and extensions. Eur. J. Oper. Res. 75, 312-331 (1994)

27. van Hoesel, C.P.M., Wagelmans, A., Wolsey, L.A.: Polyhedral characterization of the economic lotsizing problem with start-up costs. SIAM J. Discret. Math. 7, 141-151 (1994)

28. Van Vyve, M., Wolsey, L.A.: Approximate extended formulations. Math. Program. B 105, 501-522 (2006)

29. Veinott, A.F.: Minimum concave cost solution of Leontief substitution systems of multifacility inventory systems. Oper. Res. 17, 262-291 (1969)

30. Verweij, B., Wolsey, L.A.: Uncapacitated lot-sizing with buying, sales and backlogging. Optim. Methods Softw. 19, 427-436 (2004)

31. Wagner, H.M., Whitin, T.M.: Dynamic version of the economic lot size model. Manag. Sci. 5, 89-96 (1958)

32. Zangwill, W.I.: A backlogging model and a multi-echelon model of a dynamic economic lot size production system: a network approach. Manag. Sci. 15, 506-526 (1969)

33. Zhang, M., Küçükyavuz, S., Yaman, H.: A polyhedral study of multi-echelon lot sizing with intermediate demands. Oper. Res. 60, 918-936 (2012) 